\title{
Compact higher-order gas-kinetic schemes with spectral-like resolution for compressible flow simulations
}

\author{
Fengxiang Zhao ${ }^{1}$, Xing $\mathrm{Ji}^{2}$, Wei Shyy ${ }^{1}$ and Kun $\mathrm{Xu}^{1,2,3^{*}}$ (1)
}

\author{
*Correspondence: makxu@ust.hk \\ 1 Department of Mechanical and \\ Aerospace Engineering, Hong Kong \\ University of Science and \\ Technology, Clear Water Bay, \\ Kowloon, Hong Kong \\ ${ }^{2}$ Department of Mathematics, Hong \\ Kong University of Science and \\ Technology, Clear Water Bay, \\ Kowloon, Hong Kong \\ Full list of author information is \\ available at the end of the article
}

\begin{abstract}
In this paper, a class of compact higher-order gas-kinetic schemes (GKS) with spectrallike resolution will be presented. Based on the high-order gas evolution model, both the flux function and conservative flow variables in GKS can be evaluated explicitly from the time-accurate gas distribution function at a cell interface. As a result, inside each control volume both the cell-averaged flow variables and their cell-averaged gradients can be updated within each time step. The flow variable update and slope update are coming from the same physical solution at the cell interface. This strategy needs time accurate solution at a cell interface, which cannot be achieved by the Riemann problem based flow solvers, even though they can also provide the interface flux functions and interface flow variables. Instead, in order to update the slopes in the Riemann-solver based schemes, such as HWENO, there are additional governing equations for slopes or equivalent degrees of freedom inside each cell. In GKS, only a single time accurate gas evolution model is needed at the cell interface for updating cell averaged flow variables through interface fluxes and updating the cell averaged slopes through the interface flow variables. Based on both cell averaged values and their slopes, compact 6th-order and 8th-order linear and nonlinear reconstructions can be developed. As analyzed in this paper, the local linear compact reconstruction without limiter can achieve a spectral-like resolution at large wavenumber than the well-established compact scheme of Lele with globally coupled flow variables and their derivatives. For nonlinear gas dynamic evolution, in order to avoid spurious oscillation in discontinuous region, the above compact linear reconstruction from the symmetric stencil can be divided into sub-stencils and apply a biased nonlinear WENO-Z reconstruction. Consequently discontinuous solutions can be captured through the 6th-order and 8th-order compact WENO-type nonlinear reconstruction. In GKS, the time evolution solution of the gas distribution function at a cell interface is based on an integral solution of the kinetic model equation, which covers a physical process from an initial non-equilibrium state to a final equilibrium one. Since the initial non-equilibrium state is obtained based on the nonlinear WENO-Z reconstruction, and the equilibrium state is basically constructed from the linear symmetric reconstruction, the GKS evolution models unifies the nonlinear and linear reconstructions in a gas relaxation process in the determination of a time-dependent gas distribution function. This property gives GKS great advantages in capturing both discontinuous shock waves and the linear aero-acoustic waves in a (Continued on next page)
\end{abstract}


(Continued from previous page)

single computation due to its dynamical adaptation of non-equilibrium and equilibrium states in different flow regions. This dynamically adaptive model helps to solve a long lasting problem in the development of high-order schemes about the choices of the linear and nonlinear reconstructions. Compared with discontinuous Galerkin (DG) scheme, the current compact GKS uses the same local and compact stencil, achieves the 6th-order and 8th-order accuracy, uses a much larger time step with CFL number $\geq 0.3$, has the robustness as a 2 nd-order scheme, and gets accurate solutions in both shock and smooth regions without introducing trouble cell and additional limiting process. The nonlinear reconstruction in the compact GKS is solely based on the WENO-Z technique. At the same time, the current scheme solves the Navier-Stokes equations automatically due to combined inviscid and viscous flux terms from a single time evolution gas distribution function at a cell interface. Due to the use of multi-stage multi-derivative (MSMD) time-stepping technique, for achieving a 4th-order time accuracy, the current scheme uses only two stages instead of four in the traditional Runge-Kutta method. As a result, the current GKS becomes much more efficient than the corresponding same order DG method. A variety of numerical tests are presented to validate the compact 6th and 8th-order GKS. The current scheme presents a state-of-art numerical solutions under a wide range of flow conditions, i.e., strong shock discontinuity, shear instability, aero-acoustic wave propagation, and NS solutions. It promotes the development of high-order scheme to a new level of maturity. The success of the current scheme crucially depends on the high-order gas evolution model, which cannot be achieved by any other approach once the 1st-order Riemann flux function is still used in the development of high-order numerical algorithms.

Keywords: Gas-kinetic scheme, WENO reconstruction, Linear reconstruction, Compact scheme

\section{Introduction}

High-order methods with spectral resolutions and shock capturing capability are needed in many engineering applications, such as turbulent flows, aeroacoustics, and various complex flows with shock and boundary layer interactions. With the great potentials of the high-order methods in solution accuracy and computational efficiency, extensive effort has been paid on the development of high-order schemes in the past decades. However, the spectral accuracy and shock capturing seem to be contradictory in the current CFD algorithms, where the linear schemes for the smooth flow and nonlinear schemes for discontinuous flow play different roles under different flow conditions [1]. It seems hard to possess both properties in a single high-order method.

For smooth flows, the compact schemes [2-4] and discontinuous Galerkin methods (DG) or flux reconstruction (FR) [5-8] are very attractive. The compact finite difference scheme constructs implicit relation between flow variables and their derivatives from a compact stencil. Dispersion and dissipation properties of the scheme have been fully analyzed [2], and the scheme influences greatly on the development of linear schemes. The reason for designing compact scheme is its flexibility in complex geometries and effective parallelization. The DG has a second-order scheme stencil, but achieves high accuracy by using high-order piecewise polynomials within elements and evolving the multiple degrees of freedom (DOFs). Each element in DG only interacts with its neighboring elements and the scheme becomes very efficient for parallel computation [9]. Both compact and DG methods work successfully for smooth flows, and problems are still remained 
for flow simulation with shock discontinuity. Theoretically, compact scheme is physically sounded because under the CFL condition, the domain of dependence is indeed only related to the neighboring cells. All non-compact schemes must be associated with dynamical weakness because they seek for help from the cells far away which may not have any physical connection in terms of the wave propagating speed.

Nonlinear schemes have been designed for the flows with discontinuous shocks. The successful nonlinear schemes include total variation diminishing (TVD) [10], essentially non-oscillation (ENO) [11], and weighted ENO (WENO) [12, 13]. In past twenty years, the WENO-based methods have received the most attention among nonlinear schemes. The central ingredients in WENO scheme is to construct several low order polynomials and to design smoothness indicators to adaptively assemble them to get a higher one. Most current effort is about the selection of optimal stencil and the design of weighting functions. WENO scheme can achieve very high-order accuracy in the smooth region and maintain non-oscillatory property across shock waves [14]. But, their numerical dissipation is still very higher than the linear schemes $[15,16]$, and the modified WENO schemes, such as WENO-M and WENO-Z [17, 18], have been proposed recently. The hybrid linear and nonlinear schemes have been investigated as well [19-21].

In the existing DG and WENO methods, the first-order Riemann flux plays a key role for the flow evolution of compressible flow. Recently, beyond the first-order Riemann solver, many schemes based on the time-dependent high-order (more than first-order) flux functions have been developed, such as the Lax-Wendroff method [22], the generalized Riemann problem (GRP) [23-25], and ADER framework [26, 27]. An outstanding method is the two-stage fourth-order scheme for the Euler equations [28, 29], where both the flux and its time derivative are used in the construction of high-order scheme. Similar to the GRP method, the gas-kinetic scheme (GKS) is also based on the time accurate flux function at a cell interface [30-32]. The flux evaluation in GKS is based on a time evolution solution of the kinetic model equation, which provides a physical process for the gas evolution from the initial non-equilibrium state to an equilibrium one. The comparison between GRP and GKS has been presented in [33]. Under the same the multi-stage multi-derivative (MSMD) framework, based on the WENO reconstruction a two-stage fourth-order (S2O4) GKS has been successfully developed for the Euler and Navier-Stokes equations [34, 35]. The robustness of the $\mathrm{S} 2 \mathrm{O} 4$ GKS is as good as the second-order shock capturing scheme [36]. Due to the use of only two reconstructions in the 4th-order scheme, the GKS becomes very efficient in comparison with schemes based on the RungeKutta time stepping technique. Actually, the two-stage fourth-order method belongs to the MSMD framework in ODE region [37]. And it has been applied to the finite difference method and the DG method [38,39] through the direct Lax-Wendroff procedure. By combining the second-order or third-order GKS fluxes and MSMD technique again, a family of higher-order gas-kinetic methods has been constructed [40].

The time dependent gas-distribution function in GKS at the cell interface provides not only the flux and its time derivative, but also time accurate flow variables at the cell interface. The design of compact GKS based on the cell averaged and cell interface values has been conducted [41-44]. In this paper, we are going to develop a 6th-order and a 8th-order compact GKS in rectangular mesh for compressible flow simulations. The construction of the compact high-order schemes is based on the following ingredients. Firstly, the high-order gas evolution model at cell interface is used for the flux and flow variable 
evaluation. Consequently, the cell averaged flow variables and cell averaged slopes can be updated. Secondly, based on the time-dependent flux and its time derivative, the twostage fourth-order MSMD technique is used for the 4th-order temporal accuracy. Thirdly, based on the flow variables and their slopes within each cell, compact nonlinear and linear 6th-order and 8th-order spatial reconstructions will be designed for the determination of the initial non-equilibrium state and the evolved equilibrium one. Based on the GKS evolution model, in the smooth flow region the linear reconstruction will be achieved quickly in the determination of interface gas distribution function, and the spectral-like resolution can be obtained. At the same time, in the discontinuous region the nonlinear reconstruction will persist in the determination of gas evolution due to the persistence of the non-equilibrium state, and the scheme becomes robust in capturing shock and discontinuous solutions. Since the gas evolution model in GKS is based on the physical process from the initial non-equilibrium state to the final equilibrium one, the nonlinear and linear reconstructions are naturally unified in a time evolution process with automatically identified non-equilibrium and equilibrium regions. As a result, both discontinuous shocks and smooth aeroacoustic waves can be accurately captured by the current compact 6th-order and 8th-order GKS in a single computation. The compact GKS provides the Navier-Stokes solutions directly. Even for the 6th-order and 8th-order accuracy, the current compact scheme can use a time step determined by the CFL condition with a large CFL number $(\geq 0.5)$ in all test cases in this paper. The current 6th-order and 8thorder compact GKS take advantages of both nonlinear WENO-Z reconstruction for the introduction of numerical dissipation in discontinuous region and linear reconstruction with much reduced dissipation in resolving small amplitude waves. The current compact GKS are ideal schemes for the study of multiscale and complex flow interactions, such as the flow transition and turbulence.

The idea of using flow variables and their gradients has been investigated in finite volume or finite difference methods. The finite volume Hermite WENO (HWENO) schemes update both flow variables and their first derivative [45-47]. The HWENO approach is also used in the hybrid schemes [48], and a monotonicity preserving strategy for detecting troubled zones is proposed. The HWENO schemes on unstructured grids have been extensively investigated [49-51]. The HWENO approach needs additional limiters, same as the DG and reconstructed DG methods. The major advantage of these HWENO approaches is the compactness of stencils in the reconstructions. The current compact GKS is different from the HWENO approach is mainly on the update of gradients. In GKS, there is no explicit evolution equation for the gradients and the gradients are obtained rigorously from the evolution solutions at cell interfaces. Therefore, the gradients in GKS are not introduced as additional degrees of freedom with their own evolution equations. The cell-averaged gradients in GKS, similar to the cell averaged conservative variables, are obtained from the same gas distribution functions at cell interfaces. The robustness and improved resolution in GKS is due to the physically reliable gradients from the strong evolution solution of the original governing equation. In other words, the updated gradients in GKS are not coming from the "equations" by taking additional derivatives to the original ones, such as the HWENO and multi-layer compact (MLC) schemes [1]. In the HWENO and MLC, the gradients have their own evolution equations which are only reliable for the smooth flow, where new governing equations can be continuously created by taking spatial derivatives to the original equations. 
This paper is organized as follows. The GKS and MSMD method will be introduced in Section 2. In Section 3, the compact linear and nonlinear 6th-order and 8th-order reconstructions will be presented. The dissipation and dispersion will be analyzed as well. In Section 4, the compact GKS will be tested in a wide range of flow problems from the strong shock interaction to the linear acoustic wave propagation. The last section is the conclusion.

\section{Gas-kinetic scheme and multi-stage multi-derivative method}

In the past years, the gas-kinetic scheme (GKS) has been developed systematically $[30,31]$. The gas evolution model used in this paper for the flux and interface flow variable evaluation is almost identical to that of the fourth-order compact scheme [44]. Therefore, only a brief introduction about GKS will be presented in this section.

\subsection{Gas-kinetic scheme}

The gas kinetic evolution model in GKS is based on the BGK equation [52],

$$
f_{t}+\mathbf{u} \cdot \nabla f=\frac{g-f}{\tau},
$$

where $f$ is the gas distribution function, $g$ is the corresponding equilibrium state that $f$ approaches, and $\tau$ is defined as the collision time. In two-dimensional case, the equilibrium state is the Maxwellian distribution

$$
g=\rho\left(\frac{\lambda}{\pi}\right)^{\frac{K+2}{2}} e^{\lambda\left((u-U)^{2}+(v-V)^{2}+\xi^{2}\right)},
$$

where $\lambda=m / 2 k T$, and $m, k, T$ represent the molecular mass, the Boltzmann constant, and temperature, $K$ is the number of internal degrees of freedom, i.e. $K=(4-2 \gamma) /(\gamma-1)$ for two-dimensional flow, and $\gamma$ is the specific heat ratio. $\xi$ is the internal variable with $\xi^{2}=\xi_{1}^{2}+\xi_{2}^{2}+\ldots+\xi_{K}^{2}$. The collision term satisfies the following compatibility condition

$$
\int \frac{g-f}{\tau} \psi \mathrm{d} \Xi=0
$$

where $\psi=\left(\psi_{1}, \psi_{2}, \psi_{3}, \psi_{4}\right)^{T}=\left(1, u, v, \frac{1}{2}\left(u^{2}+v^{2}+\xi^{2}\right)\right), \mathrm{d} \Xi=\mathrm{d} u \mathrm{~d} v \mathrm{~d} \xi_{1} \ldots \mathrm{d} \xi_{K}$.

The macroscopic mass $\rho$, momentum $(\rho U, \rho V)$, and energy $\rho E$ at a cell interface can be evaluated from the gas distribution function,

$$
\mathbf{W}=\left(\begin{array}{c}
\rho \\
\rho U \\
\rho V \\
\rho E
\end{array}\right)=\int \psi f d \Xi,
$$

and the flux in the $x$ direction can be evaluated as well,

$$
\mathbf{F}=\int u f \psi d \Xi \text {. }
$$

Therefore, the central point in GKS is to evaluate the time-dependent gas distribution function $f$ at a cell interface.

By direct modeling on the mesh size scale [30], the conservations of mass, momentum and energy in a control volume become

$$
\frac{\mathrm{d} \mathbf{W}_{i j}}{\mathrm{~d} t}=-\frac{1}{\Delta x}\left(\mathbf{F}_{i+1 / 2, j}(t)-\mathbf{F}_{i-1 / 2, j}(t)\right)-\frac{1}{\Delta y}\left(\mathbf{G}_{i, j+1 / 2}(t)-\mathbf{G}_{i, j-1 / 2}(t)\right),
$$


where $\mathbf{W}_{i j}$ is the cell averaged conservative variables, $\mathbf{F}_{i \pm 1 / 2, j}(t)$ and $\mathbf{G}_{i, j \pm 1 / 2}(t)$ are the time dependent fluxes at cell interfaces in $x$ and $y$ directions. Like the traditional highorder finite volume scheme, the Gaussian quadrature points are used to get a high accuracy in the discretization of line integration of the flux. In this paper, three-points Gaussian quadrature will be used on each cell interface in the 2D case.

To model the interface gas distribution function, the integral solution of BGK Eq. 1 is used

$$
\begin{aligned}
f\left(x_{i+1 / 2}, y_{j \ell}, t, u, v, \xi\right)= & \frac{1}{\tau} \int_{0}^{t} g\left(x^{\prime}, y^{\prime}, t^{\prime}, u, v, \xi\right) e^{-\left(t-t^{\prime}\right) / \tau} d t^{\prime} \\
& +e^{-t / \tau} f_{0}(-u t,-v t, u, v, \xi),
\end{aligned}
$$

where $\left(x_{i+1 / 2}, y_{j_{\ell}}\right)=(0,0)$ is the location at cell interface for flux evaluation, and $x_{i+1 / 2}=$ $x^{\prime}+u\left(t-t^{\prime}\right)$ and $y_{j_{\ell}}=y^{\prime}+v\left(t-t^{\prime}\right)$ are the trajectory of particles. Here $f_{0}$ is the initial gas distribution function and $g$ is the equilibrium state in space and time. The integral solution basically states a physical process from the particle free transport in $f_{0}$ in the kinetic scale to the hydrodynamic flow evolution in the integral term of $g$. The contributions from $f_{0}$ and $g$ in the determination of $f$ at the cell interface depend on the ratio of time step to the local particle collision time, i.e., $\exp (-t / \tau)$, and the modeling of $f_{0}$ and $g$ in space and time is needed to evaluate the solution $f$ from Eq. (6). For the continuum flow simulation, such as the NS solutions, the determination of $f_{0}$ and $g$ depend only on the macroscopic flow variables and their initial reconstructions. In this paper, the WENO-Z method will be used as a nonlinear reconstruction in the determination of $f_{0}$, and the linear reconstruction is adopted in the determination of $g$. Therefore, the above integral solution not only incorporates a physical evolution process from non-equilibrium to an equilibrium state, but alo from a nonlinear reconstruction to a linear one. This fact is crucially important for the current scheme to capture both nonlinear shock and linear acoustic wave accurately in a single computation.

Based on the integral solution, a simplified third-order gas distribution function can be obtained as [53]

$$
\begin{aligned}
f\left(x_{i+1 / 2}, y_{j_{\ell}}, t, u, v, \xi\right) & =g_{0}+\bar{A} g_{0} t+\frac{1}{2} \bar{a}_{t t} g_{0} t^{2} \\
& -\tau\left[\left(\bar{a}_{1} u+\bar{a}_{2} v+\bar{A}\right) g_{0}+\left(\bar{a}_{x t} u+\bar{a}_{y t} v+\bar{a}_{t t}\right) g_{0} t\right] \\
& -e^{-t / \tau} g_{0}\left[1-\left(\bar{a}_{1} u+\bar{a}_{2} v\right) t\right] \\
& +e^{-t / \tau} g_{l}\left[1-\left(a_{1 l} u+a_{2 l} v\right) t\right] H(u) \\
& +e^{-t / \tau} g_{r}\left[1-\left(a_{1 r} u+a_{2 r} v\right) t\right](1-H(u)),
\end{aligned}
$$

where the terms related to $g_{0}$ are from the integral of the equilibrium state and the terms related to $g_{l}$ and $g_{r}$ are from the initial term $f_{0}$ in the Eq.(6). All other terms, such as $\bar{A}, \bar{a}_{t t}, \bar{a}, \ldots$, are coming from the derivatives of a Maxwellian distribution. All these coefficient in the above equation can be determined from the initially reconstructed macroscopic flow variables.

\subsection{Multi-stage multi-derivative time stepping method}

We choose the two-stage fourth-order method $[28,38]$ in the current work to achieve a fourth-order temporal accuracy. The scheme is both efficient and robust. Since the above time accurate gas distribution function at a cell interface is a complicated function of 
time in the non-smooth region, the treatment proposed in [28, 34] is used to extract the linearized flux function in time. The same idea could be used to obtain the numerical time derivative of the interface value [44] and is again used here. Theoretically, the higherorder MSMD methods could be chosen to achieve higher-order temporal accuracy [54], while the fourth-order method in time seems accurate enough in the present study.

For conservation laws, the semi-discrete finite volume scheme Eq.(5) is rewritten as

$$
\frac{\mathrm{d} \mathbf{W}_{i j}}{\mathrm{~d} t}:=\mathcal{L}\left(\mathbf{W}_{i j}\right),
$$

where $\mathcal{L}\left(\mathbf{W}_{i j}\right)$ is the numerical operator for spatial derivative of flux.

A fourth-order temporal accurate solution for $\mathbf{W}(t)$ at $t=t_{n}+\Delta t$ can be obtained by

$$
\begin{aligned}
\mathbf{W}^{*} & =\mathbf{W}^{n}+\frac{1}{2} \Delta t \mathcal{L}\left(\mathbf{W}^{n}\right)+\frac{1}{8} \Delta t^{2} \frac{\partial}{\partial t} \mathcal{L}\left(\mathbf{W}^{n}\right), \\
\mathbf{W}^{n+1} & =\mathbf{W}^{n}+\Delta t \mathcal{L}\left(\mathbf{W}^{n}\right)+\frac{1}{6} \Delta t^{2}\left(\frac{\partial}{\partial t} \mathcal{L}\left(\mathbf{W}^{n}\right)+2 \frac{\partial}{\partial t} \mathcal{L}\left(\mathbf{W}^{*}\right)\right),
\end{aligned}
$$

where $\mathcal{L}$ and $\frac{\partial}{\partial t} \mathcal{L}$ are related to the fluxes and the time derivatives of the fluxes evaluated from the time-dependent gas distribution function $f(t)$ at cell interfaces. And the middle state $\mathbf{W}^{*}$ is obtained at time $t^{*}=t^{n}+\Delta t / 2$. Again, with the time accurate gas distribution function $f(t)$, along the same line with MSMD technique the gas distribution function $f$ at a cell interface at $t^{n+1}$ becomes

$$
f^{n+1}=f^{n}+\Delta t f_{t}^{n}+\frac{1}{6} \Delta t^{2}\left(f_{t t}^{n}+2 f_{t t}^{*}\right),
$$

where $f^{*}$ is for the middle state at time $t^{*}=t^{n}+\Delta t / 2$,

$$
f^{*}=f^{n}+\frac{1}{2} \Delta t f_{t}^{n}+\frac{1}{8}(\Delta t)^{2} f_{t t}^{n} .
$$

Therefore, based on the cell interface $f_{i+1 / 2}^{n+1}$, the flow variables $\mathbf{W}_{i+1 / 2}^{n+1}$ can be explicitly obtained, i.e., $\mathbf{W}_{i+1 / 2}^{n+1}=\int \psi f_{i+1 / 2}^{n+1} d \Xi$, from which the slope inside each cell, such as in the $1 \mathrm{D}$ case, can be updated as

$$
\left(\mathbf{W}_{x}\right)_{i}^{n+1}=\left(\mathbf{W}_{i+1 / 2}^{n+1}-\mathbf{W}_{i-1 / 2}^{n+1}\right) /\left(x_{i+1 / 2}-x_{i-1 / 2}\right) .
$$

More detailed formulation for the above GKS part can be found in [44].

\section{Compact WENO reconstruction}

In the classical high-order finite volume method [11, 12], the pointwise value is reconstructed based on cell averages, and the Riemann solver is used for the flux evaluation at the interface. However, for the gas kinetic scheme, as presented in the Section 2 the cell-averaged conservative flow variables and cell-averaged slopes inside each cell can be updated. Based on these updated data, a 6th-order and a 8th-order compact linear and nonlinear reconstruction will be designed consistently in this section, which can be subsequently used in the determination of equilibrium state and initial non-equilibrium one in the gas kinetic scheme.

\subsection{Compact linear reconstruction}

In the Section 2, the conservative variables $\mathbf{W}_{i+1 / 2}$ is provided by taking moments of the gas distribution function at the cell interface. The averaged slope in the cell $I_{i}$ can be 
obtained by the Gaussian formula

$$
\mathbf{W}_{i}^{\prime}=\frac{1}{\Delta x} \int_{I_{i}} \frac{\partial \mathbf{W}}{\partial x} \mathrm{~d} x=\frac{1}{\Delta x}\left(\mathbf{W}_{i+1 / 2}-\mathbf{W}_{i-1 / 2}\right),
$$

where $\mathbf{W}_{i}^{\prime}$ is the cell averaged slope in the cell $I_{i}$.

By taking advantage of the cell averaged variables and their slopes, the compact stencil $S_{i+1 / 2}=\left\{I_{i-1}, I_{i}, I_{i+1}, I_{i+2}\right\}$ shown in Fig. 1 will be used for the reconstruction of the value at the cell interface $x_{i+1 / 2}$. On each cell $I_{i}$, the components (i.e. components of conservative or characteristic variables) of cell averages and their slopes are denoted as $Q_{i}$ and $Q_{i}^{\prime}$. Based on the large stencil $S_{i+1 / 2}$, a series of high-order polynomials can be constructed as

$$
P^{n}(x) \equiv \sum_{k=0}^{n} c_{k} x^{k}
$$

where $n$ is the order of the polynomial and $n=5,7$ correspond to the 6 th-order and 8th-order schemes, respectively.

The polynomial $P^{7}(x)$ can be uniquely determined with the condition

$$
\begin{aligned}
& \frac{1}{\Delta x} \int_{I_{k}} P^{7}(x) \mathrm{d} x=Q_{k}, \\
& \frac{1}{\Delta x} \int_{I_{k}}\left(d P^{7}(x) / d x\right) \mathrm{d} x=Q_{k}^{\prime}, I_{k} \in S_{i+1 / 2}, k=i-1, \cdots, i+2 .
\end{aligned}
$$

In order to determine the $P^{5}(x)$, the above condition has to be modified. For the cells $I_{i}$ and $I_{i+1}$, the following equations are strictly enforced

$$
\begin{aligned}
& \frac{1}{\Delta x} \int_{I_{k}} P^{5}(x) \mathrm{d} x=Q_{k}, \\
& \frac{1}{\Delta x} \int_{I_{k}}\left(d P^{5}(x) / d x\right) \mathrm{d} x=Q_{k}^{\prime}, I_{k} \in S_{i+1 / 2}, k=i, i+1,
\end{aligned}
$$

and for the cells $I_{i-1}$ and $I_{i+2}$ the following conditions are satisfied in the sense of the least square

$$
\begin{aligned}
& \frac{1}{\Delta x} \int_{I_{k}} P^{5}(x) \mathrm{d} x=Q_{k}, \\
& \frac{1}{\Delta x} \int_{I_{k}}\left(d P^{5}(x) / d x\right) \mathrm{d} x=Q_{k}^{\prime}, I_{k} \in S_{i+1 / 2}, k=i-1, i+2 .
\end{aligned}
$$

Thus the linear reconstruction $P^{n}\left(x_{i+1 / 2}\right)$ at the cell interface $x=x_{i+1 / 2}$ can be written as

$$
\begin{aligned}
P^{5}\left(x_{i+1 / 2}\right)=\frac{1}{600}\left(Q_{i-1}+299 Q_{i}+299 Q_{i+1}+Q_{i+2}-\right. \\
\left.3 \Delta x Q_{i-1}^{\prime}+111 \Delta x Q_{i}^{\prime}-111 \Delta x Q_{i+1}^{\prime}+3 \Delta x Q_{i+2}^{\prime}\right),
\end{aligned}
$$

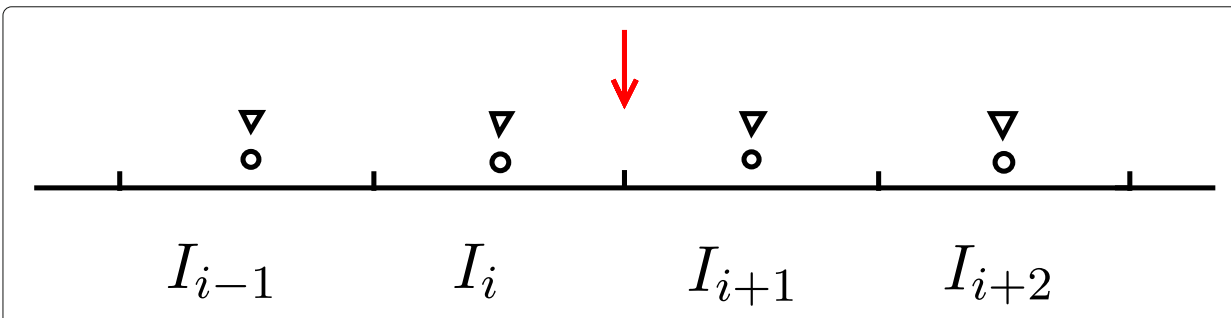

Fig. 1 The large stencil for compact reconstruction of the value at the interface $x_{i+1 / 2}$. The circles represent cell averages, and the gradients represent averaged slopes 


$$
\begin{aligned}
P^{7}\left(x_{i+1 / 2}\right)=\frac{1}{420}\left(25 Q_{i-1}+185 Q_{i}+185 Q_{i+1}+25 Q_{i+2}+\right. \\
\left.6 \Delta x Q_{i-1}^{\prime}+102 \Delta x Q_{i}^{\prime}-102 \Delta x Q_{i+1}^{\prime}-6 \Delta x Q_{i+2}^{\prime}\right),
\end{aligned}
$$

where $P^{n}\left(x_{i+1 / 2}\right)$ has the truncation error of $O\left(\Delta x^{n+1}\right)$. The first-order and second-order derivatives of the linear reconstruction at the cell interface can be obtained from $P^{n}(x)$,

$$
\begin{gathered}
P_{x}^{5}\left(x_{i+1 / 2}\right)=\frac{1}{1560 \Delta x}\left(-3 Q_{i-1}-3411 Q_{i}+3411 Q_{i+1}+3 Q_{i+2}+\right. \\
\left.11 \Delta x Q_{i-1}^{\prime}-941 \Delta x Q_{i}^{\prime}-941 \Delta x Q_{i+1}^{\prime}+11 \Delta x Q_{i+2}^{\prime}\right), \\
P_{x x}^{5}\left(x_{i+1 / 2}\right)=\frac{1}{40 \Delta x^{2}}\left(-Q_{i-1}+Q_{i}+Q_{i+1}-Q_{i+2}+\right. \\
\left.3 \Delta x Q_{i-1}^{\prime}-51 \Delta x Q_{i}^{\prime}+51 \Delta x Q_{i+1}^{\prime}-3 \Delta x Q_{i+2}^{\prime}\right), \\
P_{x}^{7}\left(x_{i+1 / 2}\right)=\frac{1}{108 \Delta x}\left(-14 Q_{i-1}-270 Q_{i}+270 Q_{i+1}+14 Q_{i+2}-\right. \\
\left.3 \Delta x Q_{i-1}^{\prime}-99 \Delta x Q_{i}^{\prime}-99 \Delta x Q_{i+1}^{\prime}-3 \Delta x Q_{i+2}^{\prime}\right), \\
P_{x x}^{7}\left(x_{i+1 / 2}\right)=\frac{1}{4 \Delta x^{2}}\left(-4 Q_{i-1}+4 Q_{i}+4 Q_{i+1}-4 Q_{i+2}-\right. \\
\left.\Delta x Q_{i-1}^{\prime}-9 \Delta x Q_{i}^{\prime}+9 \Delta x Q_{i+1}^{\prime}+\Delta x Q_{i+2}^{\prime}\right) .
\end{gathered}
$$

In order to eliminate the spurious oscillation and improve the stability, the above reconstruction is based on the characteristic variables. The dimension-by-dimension strategy is applied for the reconstruction in the two-dimensional case. The above compact reconstruction is mainly used to determine the equilibrium state in the normal direction in the gas kinetic scheme. Due to multi-dimensionality in the GKS evolution model, in the tangential direction a linear 5th-order reconstruction is used in both 6th-order and 8thorder schemes. The above linear reconstruction is used to determine the equilibrium state in GKS. In the reconstruction of the initial non-equilibrium state, the WENO-based compact nonlinear reconstruction will be designed in later subsection.

\subsection{Resolution analysis}

In this section, the resolution analysis of the above compact spatial reconstruction will be presented. The linear scalar advection equation is used for the resolution analysis,

$$
Q_{t}=-C Q_{x}
$$

where $C$ is a constant. The integral form on the cell $I_{i}$ becomes

$$
\left(Q_{i}\right)_{t}=-C\left(Q_{i}\right)_{x}
$$

where $Q_{i}$ is the cell averaged $Q(x)$ in the cell $I_{i}$.

Remark 1 In order to make Fourier expansion for the cell averages $Q_{i}$, a continuous function $\bar{Q}(x)$ is introduced [11] as follows.

$$
\bar{Q}(x)=\frac{1}{\Delta x} \int_{x-\Delta x / 2}^{x+\Delta x / 2} Q(x+y) d y .
$$

Thus the cell average $Q_{i}$ becomes the value of the continuous function $\bar{Q}(x)$ at cell center $x_{i}$.

In order to analyse the resolution of spatial discretization in the current compact schemes, we can compare the Fourier coefficients of the derivative given by the spatial 
discretization with the Fourier coefficients of exact derivative. Using the reconstructed value at the cell interface, the spatial discretization of $\left(Q_{i}\right)_{x}$ in Eq. (19) becomes

$$
\begin{aligned}
&\left(Q_{i}\right)_{x}^{6 \text { th }}=\frac{1}{600 \Delta x}( 298\left(Q_{i+1}-Q_{i-1}\right)+\left(Q_{i+2}-Q_{i-2}\right)- \\
&\left.114 \Delta x\left(Q_{i+1}^{\prime}+Q_{i-1}^{\prime}\right)+3 \Delta x\left(Q_{i+2}^{\prime}+Q_{i-2}^{\prime}\right)+222 \Delta x Q_{i}^{\prime}\right), \\
&\left(Q_{i}\right)_{x}^{8 \text { th }}=\frac{1}{420 \Delta x}\left(160\left(Q_{i+1}-Q_{i-1}\right)+25\left(Q_{i+2}-Q_{i-2}\right)-\right. \\
&\left.96 \Delta x\left(Q_{i+1}^{\prime}+Q_{i-1}^{\prime}\right)-6 \Delta x\left(Q_{i+2}^{\prime}+Q_{i-2}^{\prime}\right)+204 \Delta x Q_{i}^{\prime}\right) .
\end{aligned}
$$

For the purpose of Fourier analysis, the function $\bar{Q}(x)$ is assumed to be periodic over the domain $[0, L]$, and $\bar{Q}(x)$ can be decomposed into its Fourier coefficients

$$
\bar{Q}(x)=\sum_{k=0}^{k=N / 2} c_{k} e^{\left(\frac{2 \pi i k x}{L}\right)} .
$$

It is convenient to introduce new variables $\omega=2 \pi k \Delta x / L=2 \pi k / N$ and $s=x / \Delta x$ [2]. Then the Fourier expansion can be written as

$$
\bar{Q}(x)=\sum_{k=0}^{k=N / 2} c_{k} e^{i \omega(k) s},
$$

and the first-order derivative of $\bar{Q}(x)$ is

$$
\bar{Q}^{\prime}(x)=\sum_{k=0}^{k=N / 2} c_{k} \frac{i \omega}{\Delta x} e^{i w(k) s}=\sum_{k=0}^{k=N / 2} c_{k}^{\prime} e^{i \omega(k) s},
$$

where $c_{k}^{\prime}=c_{k} \frac{i \omega}{\Delta x}$. Here we have two further remarks for Eq. (24). Firstly, $Q_{i}^{\prime}$ is the value of the function $\bar{Q}^{\prime}(x)$ at cell center $x_{i}$. Secondly, in the compact GKS, the derivative $Q_{i}^{\prime}$ in the RHS of Eqs. (21) and (22) is the independent variables explicitly evaluated from the timeaccurate gas distribution function, which is essentially different from some other compact schemes, such as the compact schemes in $[2,4,55]$. Thus the Fourier expansion of $\bar{Q}^{\prime}(x)$ can be given theoretically if Eq. (23) is defined for the analytical solution. Suppose the numerical solution given by the compact schemes can be written as

$$
\bar{Q}_{h}(x)=\sum_{k=0}^{k=N / 2} \hat{c}_{k} e^{i \omega(k) s}, \quad \bar{Q}_{h}^{\prime}(x)=\sum_{k=0}^{k=N / 2} \hat{c}_{k}^{\prime} e^{i \omega(k) s} .
$$

Substituting the average value in Eqs. (21) and (22) with the form of Fourier expansion, we get $\hat{c}_{k}^{\prime}=i \omega^{\prime}, r t h c_{k}$, and the $\omega^{\prime}$,rth is

$$
\begin{aligned}
& \omega^{\prime}, 6 t h(\omega)=\frac{1}{300}(298 \sin (\omega)+\sin (2 \omega)-114 \omega \cos (\omega)+3 \omega \cos (2 \omega)+111 \omega), \\
& \omega^{\prime}, 8 t h(\omega)=\frac{1}{210}(160 \sin (\omega)+25 \sin (2 \omega)-96 \omega \cos (\omega)-6 \omega \cos (2 \omega)+102 \omega),
\end{aligned}
$$

where $\omega^{\prime}, r$ th is the modified wavenumber corresponding to the $r$ th-order spatial discretization. In addition to the modified wavenumber, the error in the phase speed can be alternatively used to evaluate the dispersive error. For the linear advection equation, the phase speed for a wavenumber $\omega$ is given by the current spatial discretization (exact time advancement is assumed) as

$$
\left(c_{p}\right)_{h}=\omega^{\prime}(\omega) / \omega .
$$


The comparison of the dispersion characteristics of the current compact GKS with the compact schemes by Lele [2] is shown in Fig. 2. The traditional compact schemes use only the node values [2] or cell averages [55] as independent values in spatial discretization, and the curves of modified wavenumber and phase speed in the range $\omega \in(\pi / 2, \pi)$ deviate greatly from the curves corresponding to the analytical solution. Therefore, these schemes have poor resolution for the wave with $\omega \in(\pi / 2, \pi)$, because the wavenumber range $(\pi>\omega>\pi / 2)$ corresponds to the wavelength $(2 \Delta x, 4 \Delta x)$, and such wave cannot be well determined by the cell averages (or point values) alone with less than four points in a wavelength. However, the compact GKS can retain a good resolution because of the use of slopes in each cell, and these slopes are evaluated from the gas evolution solutions at the cell interfaces which are independent from the cell averaged values. In the traditional compact schemes by Lele, the node values and derivatives are not fully independent. The coupling increases the error since the node values cannot resolve high wavenumber solution at all. As an example, we test the schemes for the initial condition $(\rho, U, p)=(1+0.2 \sin (2 \pi x), 1,1)$ in a computational domain $[0,2]$. The results of linear density wave at $t=1$ with meshes $\Delta x=1 / 2$ and $\Delta x=1 / 4$ are shown in Fig. 3 . These results demonstrate that the current compact schemes have favorable resolution in comparison with the non-compact WENO-7th-order scheme with linear reconstruction and GKS flux.

\subsection{WENO reconstruction}

In gas dynamics, shock and contact discontinuities can appear. To deal with discontinuities, WENO reconstruction $[12,13]$ can be used. In Section 3.1, the linear reconstruction gives a unique reconstructed value and its slope at the cell interface. However, in order to capture the possible discontinuity at a cell interface, the values at the left and right sides of the cell interface have to be valuated separately. Therefore, for the nonlinear reconstruction the sub-stencils have to be defined first. For simplicity, the WENO reconstruction procedure is given in detail for the construction of the left side value of the cell interface $x=x_{i+1 / 2}$. The procedure for the right side value can be obtained similarly according to the symmetric property, which will be omitted here. The left side value by WENO

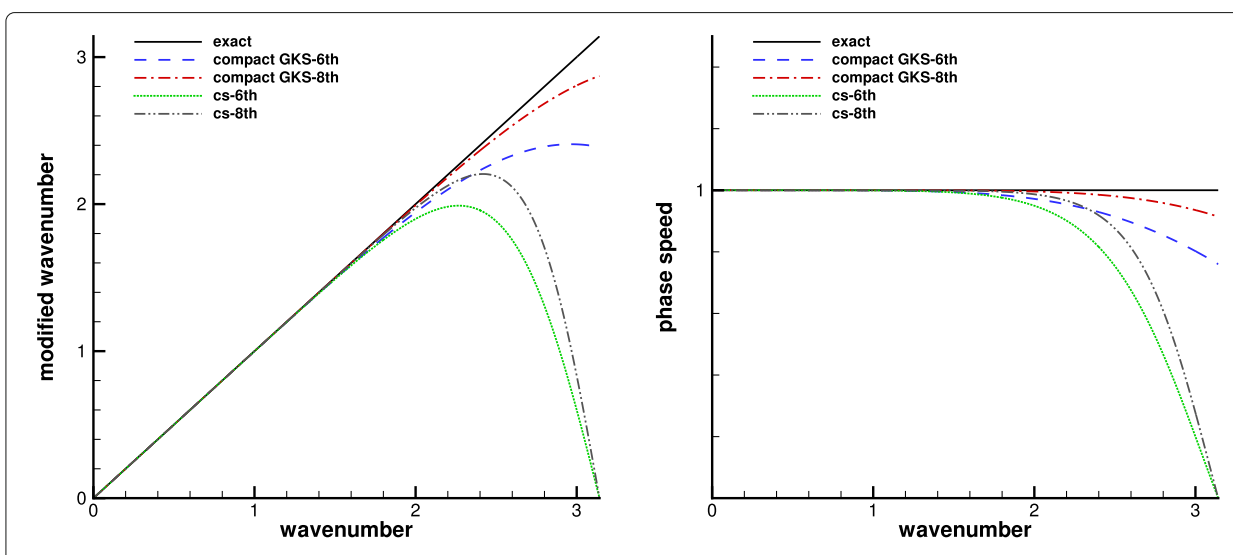

Fig. 2 Plots of modified wavenumber and phase speed vs wavenumber for different schemes. cs-6th is the compact 6th-order tridiagonal scheme studied by Lele [2], and the scheme has the best resolution in the series of 6th-order scheme; cs-8th is the compact 8th-order pentadiagonal scheme [2], and the cs-8th uses the same formula Eq. (22) in spatial discretization as the compact 8th-order GKS 


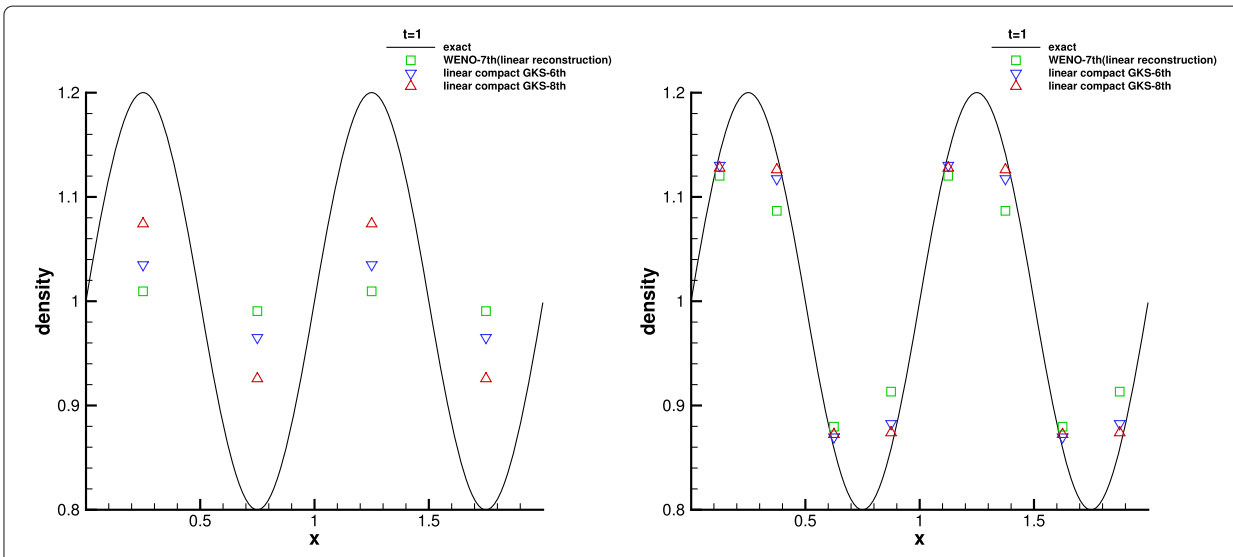

Fig. 3 Limit resolution for linear wave: Results of WENO-7th scheme with linear reconstruction and GKS flux, linear compact 6th-order and 8th-order schemes on the meshes with $\Delta x=1 / 2$ and $\Delta x=1 / 4$ at $t=1$. Since there are only two points for each wavelength on the left figure, the real "exact" solution should be averaged over half wave length as well

reconstruction is given by the candidate polynomials as follows

$$
Q_{i+1 / 2}^{n}=\sum_{k=0}^{l_{n}} \delta_{k}^{n} q_{k, i+1 / 2}^{n}, n=6,8,
$$

where $Q_{i+1 / 2}^{n}$ is the $n t h$-order WENO reconstruction for the left value of the cell interface $x_{i+1 / 2}, l_{n}$ is the number of candidate polynomials, $\delta_{k}^{n}$ is the WENO weight, and $q_{k, i+1 / 2}^{n}$ is the point value of the candidate polynomial $q_{k}^{n}(x)$ at $x_{i+1 / 2}$. In the later presentation, the sets of candidate polynomials $q_{k}^{n}(x)$ are the same for different $n t h$-order scheme, such as $n=6$ and 8 , so the superscript is omitted for simplicity.

In the current compact WENO reconstruction, three principles are considered. Firstly, if a discontinuity is located at one of the cell interfaces, theoretically the point-wise value at the interface cannot be reliable to determine the cell averaged slope on the left and right side cells. Secondly, because the smooth sub-stencils can play a dominant role in dealing with discontinuities appearing in the large stencil, the averaged slope used in the substencil should be possibly away from the interface for the data reconstruction in order to make the sub-stencil sufficiently smooth. Thirdly, the order of the candidate polynomial can be higher without increasing the spatial size of sub-stencils in the compact schemes, and such higher-order candidate polynomials can achieve better resolution to solve discontinuities without spurious oscillation. Based on the above principles, the following sub-stencils are designed (Fig. 4), where the 6th-order and 8th-order reconstructions are based on the same sub-stencils,

$$
\begin{aligned}
& S_{0}=\left\{Q_{i-1}, Q_{i}, Q_{i-1}^{\prime}\right\} \leftrightarrow q_{0}(x), \\
& S_{1}=\left\{Q_{i-1}, Q_{i}, Q_{i+1}\right\} \leftrightarrow q_{1}(x), \\
& S_{2}=\left\{Q_{i}, Q_{i+1}, Q_{i+2}, Q_{i+2}^{\prime}\right\} \leftrightarrow q_{2}(x), \\
& S_{3}=\left\{Q_{i-1}, Q_{i}, Q_{i+1}\right\} \leftrightarrow q_{3}(x), \\
& S_{4}=\left\{Q_{i}, Q_{i+1}, Q_{i+2}\right\} \leftrightarrow q_{4}(x), \\
& S_{5}=\left\{Q_{i-1}, Q_{i}, Q_{i+1}, Q_{i-1}^{\prime}, Q_{i}^{\prime}\right\} \leftrightarrow q_{5}(x), \\
& S_{6}=\left\{Q_{i}, Q_{i+1}, Q_{i+2}, Q_{i+1}^{\prime}, Q_{i+2}^{\prime}\right\} \leftrightarrow q_{6}(x) .
\end{aligned}
$$




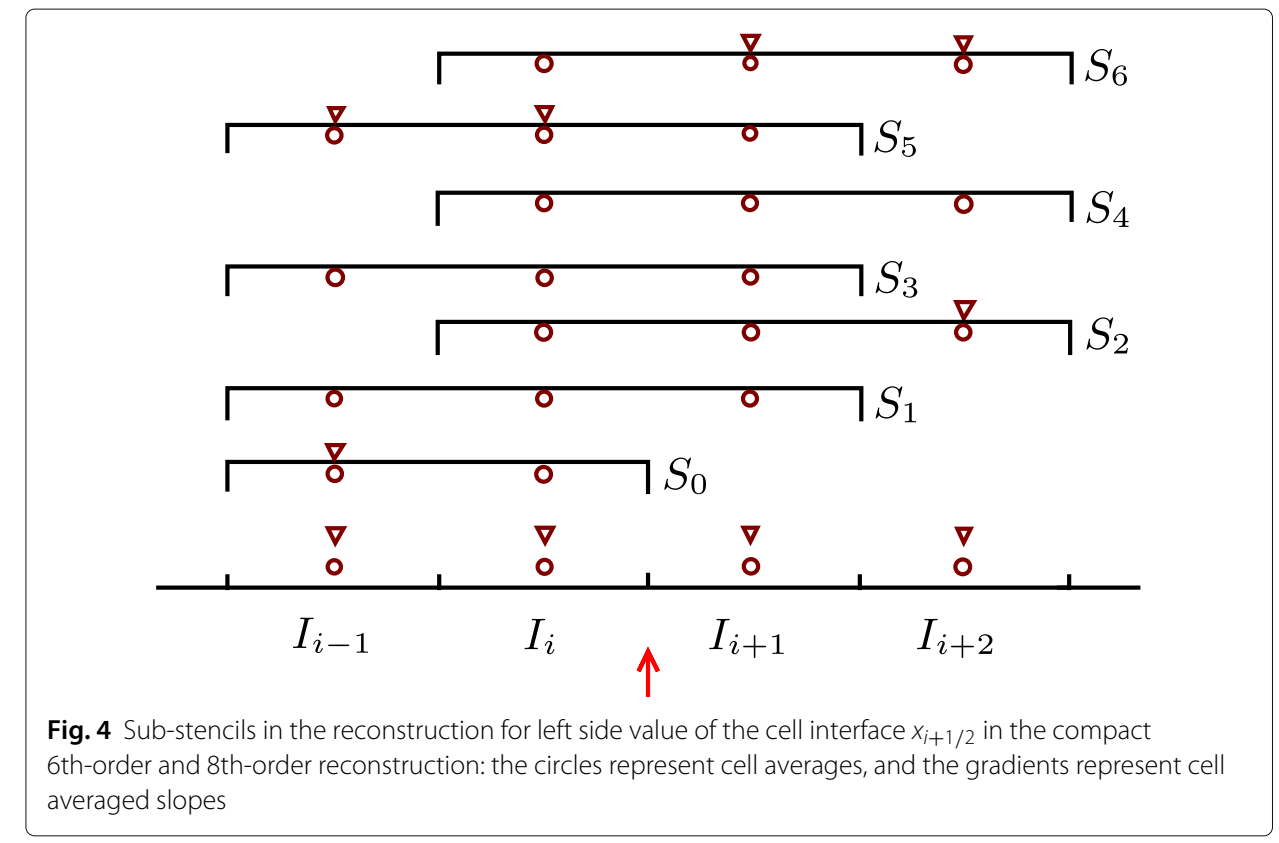

$q_{2}(x)$ is a cubic polynomial, $q_{5}(x)$ and $q_{6}(x)$ are fourth-order polynomials, and others are quadratic polynomials. According to the similar reconstruction conditions in Eq. (11), $q_{k, i+1 / 2}$ can be obtained as

$$
\left\{\begin{array}{l}
q_{0, i+1 / 2}=\frac{1}{6}\left(-7 Q_{i-1}+13 Q_{i}-4 h Q_{i-1}^{\prime}\right) \\
q_{1, i+1 / 2}=\frac{1}{6}\left(-Q_{i-1}+5 Q_{i}+2 Q_{i+1}\right), \\
q_{2, i+1 / 2}=\frac{1}{24}\left(5 Q_{i}+32 Q_{i+1}-13 Q_{i+2}+6 h Q_{i+2}^{\prime}\right), \\
q_{3, i+1 / 2}=\frac{1}{6}\left(-Q_{i-1}+5 Q_{i}+2 Q_{i+1}\right), \\
q_{4, i+1 / 2}=\frac{1}{6}\left(2 Q_{i}+5 Q_{i+1}-Q_{i+2}\right), \\
q_{5, i+1 / 2}=\frac{1}{30}\left(10 Q_{i-1}+19 Q_{i}+Q_{i+1}+3 h Q_{i-1}^{\prime}+21 h Q_{i}^{\prime}\right) \\
q_{6, i+1 / 2}=\frac{1}{30}\left(Q_{i}+19 Q_{i+1}+10 Q_{i+2}-21 h Q_{i+1}^{\prime}-3 h Q_{i+2}^{\prime}\right)
\end{array}\right.
$$

In the smooth region, the convex combination with $\delta_{k}^{n}=d_{k}^{n}$ recovers the reconstruction in Eqs. (14) and (15), which can be the condition to get the linear weights [13]. The linear weight $d_{k}^{6}$ of the 6th-order reconstruction can be obtained as

$$
d_{0}^{6}=\frac{33}{700}, d_{1}^{6}=\frac{11}{140}, d_{2}^{6}=\frac{22}{175}, d_{3}^{6}=\frac{11}{100}, d_{4}^{6}=\frac{11}{100}, d_{5}^{6}=\frac{37}{140}, d_{6}^{6}=\frac{37}{140},
$$

and the $d_{k}^{8}$ of 8th-order reconstruction are

$$
d_{0}^{8}=\frac{3}{98}, d_{1}^{8}=\frac{5}{98}, d_{2}^{8}=\frac{4}{49}, d_{3}^{8}=\frac{7}{98} d_{4}^{8}=\frac{7}{98}, d_{5}^{8}=\frac{17}{49}, d_{6}^{8}=\frac{17}{49} .
$$


The WENO-Z nonlinear weights are used in the current compact schemes and they are defined as [18]

$$
\delta_{k}^{n}=\frac{\alpha_{k}^{n}}{\sum_{m=0}^{l_{n}} \alpha_{m}^{n}}, \alpha_{k}^{n}=d_{k}^{n}\left[1+\left(\frac{Z_{r e f}^{n}}{\beta_{k}+\epsilon}\right)\right], k=0, \ldots, l_{n},
$$

where $Z_{r e f}^{n}$ is the local higher-order reference value, which is related to the accuracy of the scheme and is given in detail later. Here $\beta_{k}$ is the smooth indicator and defined as [13]

$$
\beta_{k}=\sum_{r=1}^{r_{k}} \Delta x^{2 r-1} \int_{x_{i-1 / 2}}^{x_{i}+1 / 2}\left(\frac{\mathrm{d}^{r}}{\mathrm{~d} x^{r}} q_{k}(x)\right)^{2} d x,
$$

where $r_{k}$ is the order of $q_{k}(x)$.

In smooth region, the first two candidate polynomial $q_{0}(x)$ and $q_{1}(x)$ can be combined into a cubic polynomial which is symmetric counterpart of $q_{2}(x)$. Then, current sub-stencils can become basically symmetric for interface $x_{i+1 / 2}$. In order to maintain the symmetry for the nonlinear schemes, the smooth indicator $\beta_{1}$ of $q_{1}(x)$ corresponding to $S_{1}$ is replaced by the indicator of the cubic polynomial $\widetilde{q}_{1}(x)$ on $\widetilde{S}_{1}=$ $\left\{Q_{i-1}, Q_{i}, Q_{i+1}, Q_{i-1}^{\prime}\right\}$. Even without showing in this paper, some tests demonstrate that the current choice $\beta_{1}$ can present a better resolution in the numerical results with excellent robustness. The detailed formulae for all $\beta_{k}, k=0, \ldots, l_{n}$ are given in Appendix.

\subsection{Accuracy of the compact reconstruction}

In this subsection, the accuracy of the compact reconstruction is analysed. The nonlinear reconstruction in Eq. (28) can be rewritten as

$$
Q_{i+1 / 2}^{n}=\sum_{k=0}^{l_{n}} d_{k}^{n} q_{k, i+1 / 2}+\sum_{k=0}^{l_{n}}\left(\delta_{k}^{n}-d_{k}^{n}\right) q_{k, i+1 / 2} .
$$

The reconstruction can be split into two terms [17], i.e., the linear term and nonlinear term. For the linear part $Q_{i+1 / 2}^{n, o p t} \equiv \sum_{k=0}^{l_{n}} d_{k}^{n} q_{k, i+1 / 2}$, the error can be given as

$$
Q_{i+1 / 2}^{n, o p t}-Q\left(x_{i+1 / 2}\right)=B^{n}\left(x_{i+1 / 2}\right) \Delta x^{n}+O\left(\Delta x^{n+1}\right),
$$

where $Q\left(x_{i+1 / 2}\right)$ is the exact value at $x=x_{i+1 / 2}$. In the second term in Eq. (32), the point value $q_{k, i+1 / 2}$ approximates $Q\left(x_{i+1 / 2}\right)$ as follows

$$
q_{k, i+1 / 2}=Q\left(x_{i+1 / 2}\right)+B_{k}\left(x_{i+1 / 2}\right) \Delta x^{r_{k}}+O\left(\Delta x^{r_{k}+1}\right) .
$$

Substituting $q_{k, i+1 / 2}$ into Eq. (32) and taking $\sum_{k=0}^{l_{n}} \delta_{k}^{n}=\sum_{k=0}^{l_{n}} d_{k}^{n}=1$ into account, we have

$$
\begin{aligned}
Q_{i+1 / 2}^{n} & =Q_{i+1 / 2}^{n, o p t}+\sum_{k=0}^{l_{n}}\left(\delta_{k}^{n}-d_{k}^{n}\right)\left(Q\left(x_{i+1 / 2}\right)+B_{k}\left(x_{i+1 / 2}\right) \Delta x^{r_{k}}+O\left(\Delta x^{r_{k}+1}\right)\right) \\
& =Q_{i+1 / 2}^{n, o p t}+\sum_{k=0}^{l_{n}} B_{k}\left(x_{i+1 / 2}\right)\left(\delta_{k}^{n}-d_{k}^{n}\right) \Delta x^{r_{k}}+\sum_{k=0}^{l_{n}}\left(\delta_{k}^{n}-d_{k}^{n}\right) O\left(\Delta x^{r_{k}+1}\right) .
\end{aligned}
$$

To achieve the formal order of accuracy for the 6th-order and 8th-order reconstructions, the following sufficient condition is proposed.

$$
\delta_{k}^{n}-d_{k}^{n}=O\left(\Delta x^{n-3+n^{\prime}}\right), n^{\prime} \geq 0 .
$$

For the current nonlinear weights with WENO-Z weighting functions, with the following formulation of the local higher-order reference value $Z_{r e f}^{n}$, the sufficient condition 
Eq. (33) can be satisfied when

$$
Z_{r e f}^{n}=\left|3\left(\beta_{0}-\beta_{4}\right)+\left(\beta_{4}-\beta_{3}\right)\right| .
$$

In order to prove that the sufficient condition can be met, according to the Taylor series of $\beta_{k}, k=0,3,4$,

$$
\begin{aligned}
& \beta_{0}=\left(\left(Q_{i}^{(1)}\right)^{2}+\frac{13}{12}\left(Q_{i}^{(2)} \Delta x\right)^{2}\right) \Delta x^{2}-\frac{1}{3} Q_{i}^{(1)} Q_{i}^{(3)} \Delta x^{4}+J_{0}\left(Q_{i}^{(l)}, \Delta x\right) \Delta x^{5}, \\
& \beta_{3}=\left(\left(Q_{i}^{(1)}\right)^{2}+\frac{13}{12}\left(Q_{i}^{(2)} \Delta x\right)^{2}\right) \Delta x^{2}+\frac{1}{3} Q_{i}^{(1)} Q_{i}^{(3)} \Delta x^{4}+J_{3}\left(Q_{i}^{(l)}, \Delta x\right) \Delta x^{5}, \\
& \beta_{4}=\left(\left(Q_{i}^{(1)}\right)^{2}+\frac{13}{12}\left(Q_{i}^{(2)} \Delta x\right)^{2}\right) \Delta x^{2}-\frac{2}{3} Q_{i}^{(1)} Q_{i}^{(3)} \Delta x^{4}+J_{4}\left(Q_{i}^{(l)}, \Delta x\right) \Delta x^{5}, l=1,2, \cdots
\end{aligned}
$$

we have

$$
Z_{\text {ref }}^{n}=\left|3 J_{0}-J_{3}-2 J_{4}\right| \Delta x^{5},
$$

where $J_{0}, J_{3}$ and $J_{4}$ are the functions of the Taylor expanded terms of $\bar{Q}(x)$ as $\bar{Q}(x)$ is always continuous. Suppose $O\left(J_{k}\right) \sim O(1)$, the sufficient condition Eq. (33) is satisfied by

$$
\begin{aligned}
\alpha_{k}^{n} & =d_{k}^{n}\left[1+\left(\frac{Z_{r e f}^{n}}{\beta_{k}+\epsilon}\right)\right]=d_{k}^{n}\left[C+O\left(\Delta x^{5}\right)\right], k=0, \ldots, l_{n}, \\
\delta_{k}^{n} & =\frac{\alpha_{k}^{n}}{\sum_{m=0}^{l_{n}} \alpha_{m}^{r}}=d_{k}^{n}+O\left(\Delta x^{5}\right),
\end{aligned}
$$

where $C \geq 1$ is a positive constant.

\section{Numerical tests}

In this section, we are going to test the 6th-order and 8th-order compact gas kinetic schemes. The cases include linear acoustic waves, blast wave, shock-shock interactions, shock acoustic wave interaction, as well as viscous flow computations. The mesh used in this paper is rectangular one and the time step is determined by the CFL condition with a CFL number $(\geq 0.3)$ in all test cases. In all tests, the same linear reconstruction is used for the equilibrium state and the nonlinear reconstruction for the non-equilibrium state. There is no any additional "trouble cell" detection or any additional limiter designed for specific test. The gas kinetic evolution model basically presents a dynamical process from the nonlinear to linear one, and the convergence rate $\exp (-\Delta t / \tau)$ depends on the flow condition. The collision time $\tau$ for inviscid flow at a cell interface is defined by

$$
\tau=\epsilon \Delta t+C\left|\frac{p_{l}-p_{r}}{p_{l}+p_{r}}\right| \Delta t
$$

where $\varepsilon=0.01, C=1$, and $p_{l}$ and $p_{r}$ are the pressures at the left and right sides of a cell interface. For the viscous flow, the collision time is related to the viscosity coefficient,

$$
\tau=\frac{\mu}{p}+C\left|\frac{p_{l}-p_{r}}{p_{l}+p_{r}}\right| \Delta t
$$

where $\mu$ is the dynamic viscosity coefficient and $p$ is the pressure at the cell interface. In smooth flow regions, it will reduce to $\tau=\mu / p$. The reason for including pressure jump term in the particle collision time is to add artificial dissipation in the discontinuous region to keep the non-equilibrium dynamics in the shock layer. 


\subsection{Accuracy tests}

The one-dimensional advection of density perturbation is tested first, and the initial conditions are given as follows

$$
\rho(x)=1+0.2 \sin (\pi x), \quad U(x)=1, \quad p(x)=1, x \in[0,2] .
$$

The periodic boundary condition is adopted, and the analytic solutions are

$$
\rho(x, t)=1+0.2 \sin (\pi(x-t)), \quad U(x, t)=1, \quad p(x, t)=1 .
$$

With the $r$ th-order spatial reconstruction and 2-stage 4th-order temporal discretization, the leading term of the truncation error is $O\left(\Delta x^{r}+\Delta t^{4}\right)$. To keep the $r$ th-order accuracy in the test, $\Delta t=C \Delta x^{r / 4}$ needs to be used for the $r$ th-order scheme. In the computation, the uniform meshes with $N$ mesh points are used. The $L^{1}, L^{2}$ and $L^{\infty}$ errors and convergence orders at $t=2$ for the 6th-order and 8th-order compact GKS are presented in Tables 1, 2, 3 and 4, respectively. For the 8th-order scheme, the errors reduce quickly, and the order of accuracy does not attain 8 with $N=80$ due to the limited round-off error.

\subsection{One-dimensional Riemann problems}

To test the performance of the schemes for capturing high frequency waves, the ShuOsher problem for density-wave shock interaction is tested [56]. The initial condition is given by

$$
(\rho, U, p)= \begin{cases}(3.857134,2.629369,10.33333), & x \leq 1, \\ (1+0.2 \sin (5 x), 0,1), & 1<x \leq 10 .\end{cases}
$$

The computational domain is $[0,10]$ and 200 uniform mesh points are used. The nonreflecting boundary condition is used at both ends. The computed density profile and local enlargement at $t=1.8$ from different schemes are shown in Fig. 5. Current compact 6th-order and 8th-order schemes perform well with such a coarse mesh. Due to the improvement of order of accuracy, the 8th-order scheme performs better then the 6th-order one.

As an extension of the Shu-Osher problem, the Titarev-Toro problem is tested as well [57], and the initial condition in this case is the following

$$
(\rho, U, p)= \begin{cases}(1.515695,0.523346,1.805), & -5<x \leq-4.5, \\ (1+0.1 \sin (20 \pi x), 0,1), & -4.5<x<5 .\end{cases}
$$

The computational domain is $[-5,5]$. The non-reflecting boundary condition is imposed on left end, and the fixed wave profile is given on the right end. Both compact 6th-order and 8th-order schemes are tested for this case. The computed density profile

Table 1 1-D accuracy test: errors and convergence orders of 6th-order compact linear scheme with $\Delta t=0.3 \Delta x^{3 / 2}$

\begin{tabular}{lllllll}
\hline Mesh length & $L^{1}$ error & Order & $L^{2}$ error & Order & $L^{\infty}$ error & Order \\
\hline $1 / 5$ & $1.761 \mathrm{e}-003$ & & $1.976 \mathrm{e}-003$ & & $2.721 \mathrm{e}-003$ & \\
$1 / 10$ & $3.171 \mathrm{e}-005$ & 5.80 & $3.527 \mathrm{e}-005$ & 5.81 & $4.899 \mathrm{e}-005$ & 5.80 \\
$1 / 20$ & $5.017 \mathrm{e}-007$ & 5.98 & $5.554 \mathrm{e}-007$ & 5.99 & $7.803 \mathrm{e}-007$ & 5.97 \\
$1 / 40$ & $7.818 \mathrm{e}-009$ & 6.00 & $8.676 \mathrm{e}-009$ & 6.00 & $1.224 \mathrm{e}-008$ & 5.99 \\
$1 / 80$ & $1.220 \mathrm{e}-010$ & 6.00 & $1.355 \mathrm{e}-010$ & 6.00 & $1.915 \mathrm{e}-010$ & 6.00 \\
\hline
\end{tabular}


Table 2 1-D accuracy test: errors and convergence orders of 8th-order compact linear scheme with $\Delta t=0.3 \Delta x^{2}$

\begin{tabular}{lllllll}
\hline Mesh length & $L^{1}$ error & Order & $L^{2}$ error & Order & $L^{\infty}$ error & Order \\
\hline $1 / 5$ & $1.196 \mathrm{e}-004$ & & $1.344 \mathrm{e}-004$ & & $1.849 \mathrm{e}-004$ & \\
$1 / 10$ & $4.715 \mathrm{e}-007$ & 7.99 & $5.301 \mathrm{e}-007$ & 7.99 & $7.286 \mathrm{e}-007$ & 7.99 \\
$1 / 20$ & $1.806 \mathrm{e}-009$ & 8.03 & $1.998 \mathrm{e}-009$ & 8.05 & $2.798 \mathrm{e}-009$ & 8.02 \\
$1 / 40$ & $6.979 \mathrm{e}-012$ & 8.02 & $7.744 \mathrm{e}-012$ & 8.01 & $1.092 \mathrm{e}-011$ & 8.00 \\
$1 / 80$ & $5.588 \mathrm{e}-014$ & 6.96 & $6.207 \mathrm{e}-014$ & 6.96 & $9.614 \mathrm{e}-014$ & 6.83 \\
\hline
\end{tabular}

with 1000 mesh points at $t=5$, local enlargement, and the exact solution for the TitarevToro problem are shown in Fig. 6. In order to show the importance and accuracy of the 6th-order and 8th-order compact reconstructions, the results from the non-compact 7thorder WENO-JS reconstruction based GKS, which uses the cell-averaged flow variables only, are included as well. As shown in Fig. 6, even with 7th-order WENO reconstruction the dissipation and dispersion errors are much larger than those from the current 6th-order compact GKS. Based on this observation, it clearly indicates that the use of high-order evolution model for the update of slope is favorable in the design of high-order schemes. The compactness of the scheme is also physically sounded because the CFL condition constrains the signal propagation locally to the neighboring cells only within a time step. To design a reliable compact scheme depends on the high-order gas evolution model at the cell interface. Any scheme based on the first order Riemann solver cannot achieve such a goal for a scheme with the properties of compactness, large CFL number, robustness, and high efficiency.

The third test is the Woodward-Colella blast wave problem [58], and the initial conditions are given as follows

$$
(\rho, U, p)= \begin{cases}(1,0,1000), & 0 \leq x<10 \\ (1,0,0.01), & 10 \leq x<90 \\ (1,0,100), & 90 \leq x \leq 100\end{cases}
$$

The computational domain is $[0,100]$. The reflecting boundary conditions are imposed on both ends. The density distributions and local enlargement from the 6th-order and 8th-order compact GKS are presented in Fig. 7 at $t=3.8$ with 200 and 400 mesh points.

\subsection{One-dimensional acoustic wave}

The one-dimensional acoustic wave propagation in $x$-direction was proposed by Bai et al. in [1]. The test demonstrates the high order and high resolution of the compact GKS to compute acoustic wave propagating through a long-distance. The initial conditions are

Table 3 1-D accuracy test: errors and convergence orders of 6th-order compact nonlinear schemes with $\Delta t=0.3 \Delta x^{3 / 2}$

\begin{tabular}{lllllll}
\hline mesh length & $L^{1}$ error & Order & $L^{2}$ error & Order & $L^{\infty}$ error & Order \\
\hline $1 / 5$ & $7.495 \mathrm{e}-003$ & & $8.448 \mathrm{e}-003$ & & $1.248 \mathrm{e}-002$ & \\
$1 / 10$ & $3.624 \mathrm{e}-004$ & 4.37 & $4.307 \mathrm{e}-004$ & 4.29 & $7.357 \mathrm{e}-004$ & 4.08 \\
$1 / 20$ & $7.877 \mathrm{e}-006$ & 5.52 & $8.677 \mathrm{e}-006$ & 5.63 & $1.461 \mathrm{e}-005$ & 5.65 \\
$1 / 40$ & $4.216 \mathrm{e}-008$ & 7.55 & $5.440 \mathrm{e}-008$ & 7.32 & $1.235 \mathrm{e}-007$ & 6.89 \\
$1 / 80$ & $2.119 \mathrm{e}-010$ & 7.64 & $2.647 \mathrm{e}-010$ & 7.68 & $7.407 \mathrm{e}-010$ & 7.38 \\
$1 / 160$ & $2.100 \mathrm{e}-012$ & 6.66 & $2.279 \mathrm{e}-012$ & 6.86 & $3.540 \mathrm{e}-012$ & 7.71 \\
\hline
\end{tabular}


Table 4 1-D accuracy test: errors and convergence orders of 8th-order compact nonlinear scheme with $\Delta t=0.3 \Delta x^{2}$

\begin{tabular}{lllllll}
\hline Mesh length & $L^{1}$ error & Order & $L^{2}$ error & Order & $L^{\infty}$ error & Order \\
\hline $1 / 5$ & $5.154 \mathrm{e}-003$ & & $5.969 \mathrm{e}-003$ & & $8.959 \mathrm{e}-003$ & \\
$1 / 10$ & $3.825 \mathrm{e}-004$ & 3.75 & $4.392 \mathrm{e}-004$ & 3.76 & $6.522 \mathrm{e}-004$ & 3.78 \\
$1 / 20$ & $7.963 \mathrm{e}-006$ & 5.59 & $9.252 \mathrm{e}-006$ & 5.57 & $1.588 \mathrm{e}-005$ & 5.36 \\
$1 / 40$ & $4.250 \mathrm{e}-008$ & 7.55 & $6.042 \mathrm{e}-008$ & 7.26 & $1.503 \mathrm{e}-007$ & 6.72 \\
$1 / 80$ & $1.339 \mathrm{e}-010$ & 8.31 & $2.201 \mathrm{e}-010$ & 8.10 & $7.984 \mathrm{e}-010$ & 7.56 \\
$1 / 160$ & $2.827 \mathrm{e}-013$ & 8.89 & $3.801 \mathrm{e}-013$ & 9.18 & $1.206 \mathrm{e}-012$ & 9.37 \\
\hline
\end{tabular}

given as follows

$$
\begin{aligned}
& U=U_{\infty}+\delta U, \delta U=\epsilon a_{\infty} \cos (\omega x), U_{\infty}=0 \\
& \rho=\rho_{\infty}+\delta \rho, \delta \rho=\epsilon \rho_{\infty} \cos (2 \omega x), \rho_{\infty}=1.1771 \\
& \frac{p}{p_{\infty}}=\left(\frac{\rho}{\rho_{\infty}}\right)^{r}, p_{\infty}=101325.0 \\
& a_{\infty}=\sqrt{\gamma \frac{p_{\infty}}{\rho_{\infty}}},
\end{aligned}
$$

where $\epsilon=10^{-5}$ is the magnitude of initial perturbation, and $\omega=6 \pi$ is the wavenumber of initial perturbations in velocity. The specific heat ratio is $\gamma=1.4$. The acoustic wave given above is approximately linear because of the very small $\epsilon$, and an analytical solution [1] is given from the approximate acoustic wave equation,

$$
\begin{aligned}
\rho(x, t)= & \rho_{\infty}+\frac{1}{2} \epsilon \rho_{\infty}\left[\cos \left(2 \omega\left(x-a_{\infty} t\right)\right)+\cos \left(2 \omega\left(x+a_{\infty} t\right)\right)+\right. \\
& \left.\cos \left(\omega\left(x-a_{\infty} t\right)\right)-\cos \left(\omega\left(x+a_{\infty} t\right)\right)\right], \\
U(x, t)= & \frac{1}{2} \epsilon a_{\infty}\left[\cos \left(2 \omega\left(x-a_{\infty} t\right)\right)-\cos \left(2 \omega\left(x+a_{\infty} t\right)\right)+\right. \\
& \left.\cos \left(\omega\left(x-a_{\infty} t\right)\right)+\cos \left(\omega\left(x+a_{\infty} t\right)\right)\right], \\
p(x, t)= & p_{\infty}+\frac{1}{2} \gamma \epsilon p_{\infty}\left[\cos \left(2 \omega\left(x-a_{\infty} t\right)\right)+\cos \left(2 \omega\left(x+a_{\infty} t\right)\right)+\right. \\
& \left.\cos \left(\omega\left(x-a_{\infty} t\right)\right)-\cos \left(\omega\left(x+a_{\infty} t\right)\right)\right] .
\end{aligned}
$$
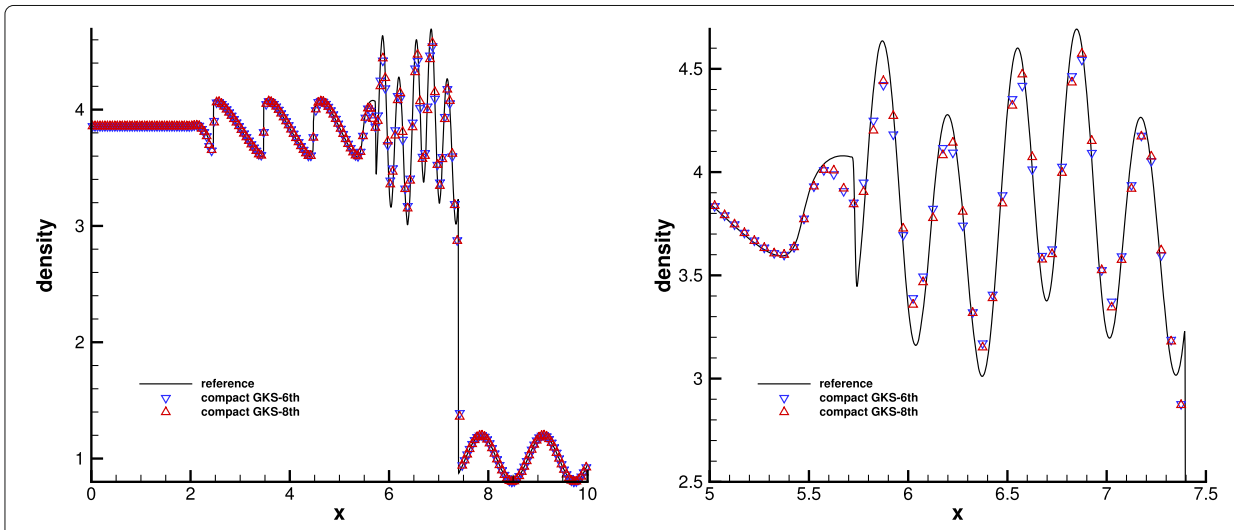

Fig. 5 Shu-Osher problem: the density distribution and local enlargement by 6th-order and 8th-order compact GKS at $t=1.8$ with 200 mesh points 


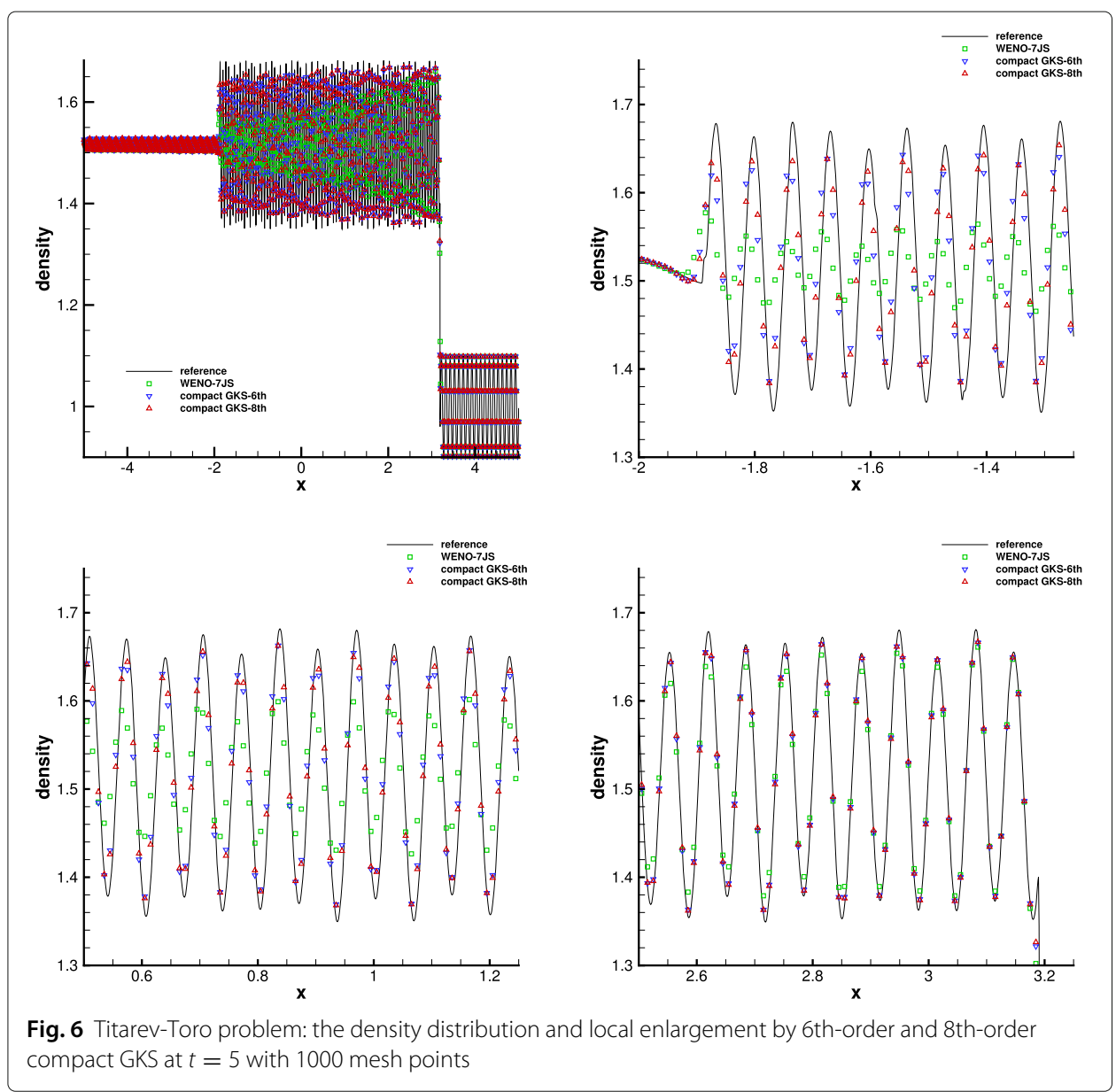

The computational domain is $[0,1 / 3]$. Periodic boundary conditions on both sides are adopted.

When giving the initial conditions in the computation, we first obtain the cell average of the primitive variables, i.e. $\bar{Q}_{i}^{p r}=\int_{i-1 / 2}^{i+1 / 2} Q^{p r}(x) d x$ exactly, where $Q^{p r}=(\rho, U, p)$. Then convert them to the conservative variables. Similarly, we could obtain the cell average of the derivatives, i.e. $\bar{Q}_{x, i}^{p r}=\int_{i-1 / 2}^{i+1 / 2} Q_{x}^{p r}(x) d x$ exactly, from which the derivatives of conservative variables can be obtained by the chain rules. Theoretically, this has a 2nd-order accuracy in space. However, it is enough for the comparison since the analytical solution in Eq. (35) is also an approximate solution. Besides the 6th-order and 8th-order compact GKS, a compact linear GKS, where the initial reconstruction for $f_{0}$ is from the linear reconstruction and has no shock capturing property, will be tested as well.

Figures 8 and 9 show the density distributions at $t=0.01$ (left) and $t=1.0$ (right) with 20 and 40 uniform mesh points. The computational time is greatly larger than $\lambda_{\rho_{0}} / a_{\infty}=4.8 \times 10^{-4}$, where $\lambda_{\rho_{0}}$ is the initial wavelength of density perturbation. Thus the distance of acoustic wave propagation is greatly larger than the initial wavelength of density perturbation. The results from GKS with linear and nonlinear reconstructions are in good agreement. The results with 40 mesh points agree well with the analytical solution, where the small deviation near the extreme point is caused by the approximate analytical solution [1]. This case also shows that the 6th-order GKS cannot give the 

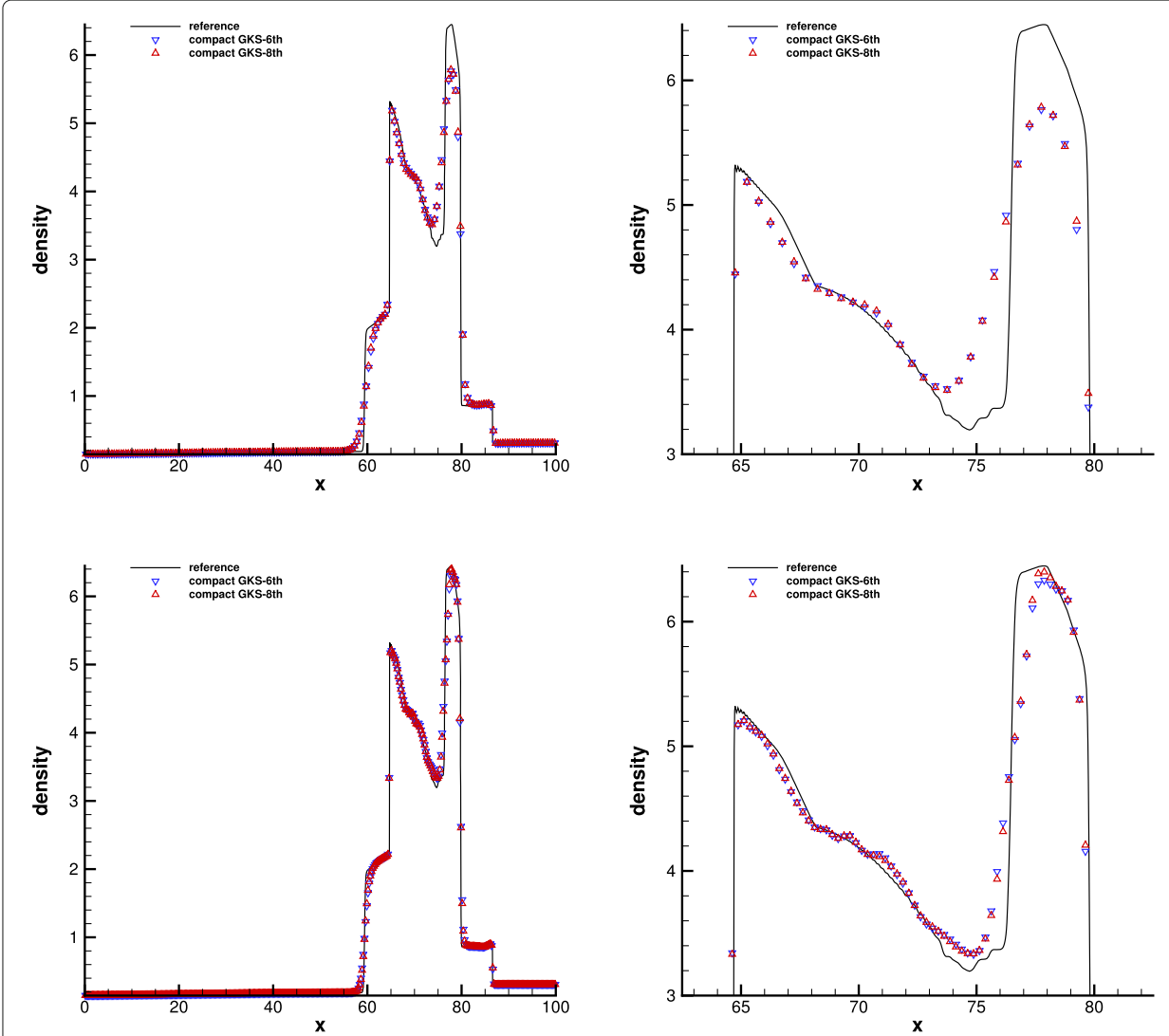

Fig. 7 Blast wave problem: the density distributions from 6th-order and 8th-order compact GKS at $t=3.8$ with 200 (up) and 400 (down) mesh points

accurate solution with 20 mesh points after a long time wave propagation (100 periods) due to the relative large numerical error in comparison with the 8th-order scheme. The results indicate that the current GKS, even with shock capturing property, has no much difference in performance in comparison with the purely linear scheme in the acoustic wave case.
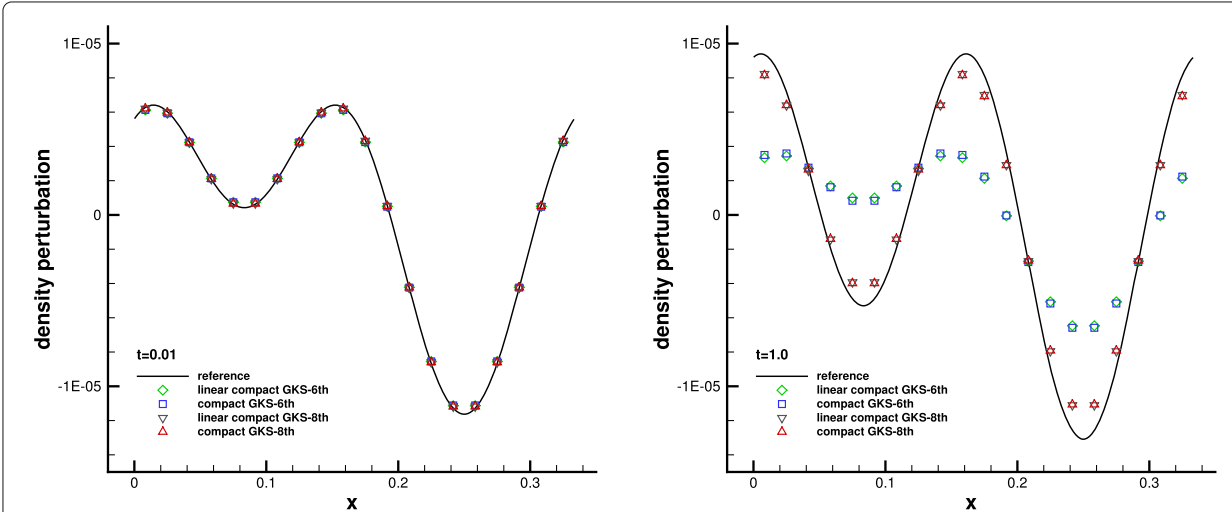

Fig. 8 One-dimensional acoustic problem: the distributions of density perturbation obtained by compact GKS at $t=0.01$ (left, 1 period) and $t=1.0$ (right, 100 periods) with 20 mesh points. The ratio of non-dimensional initial density wavelength to sound speed is $\lambda_{\rho_{0}} / a_{\infty}=4.8 \times 10^{-4}$ 

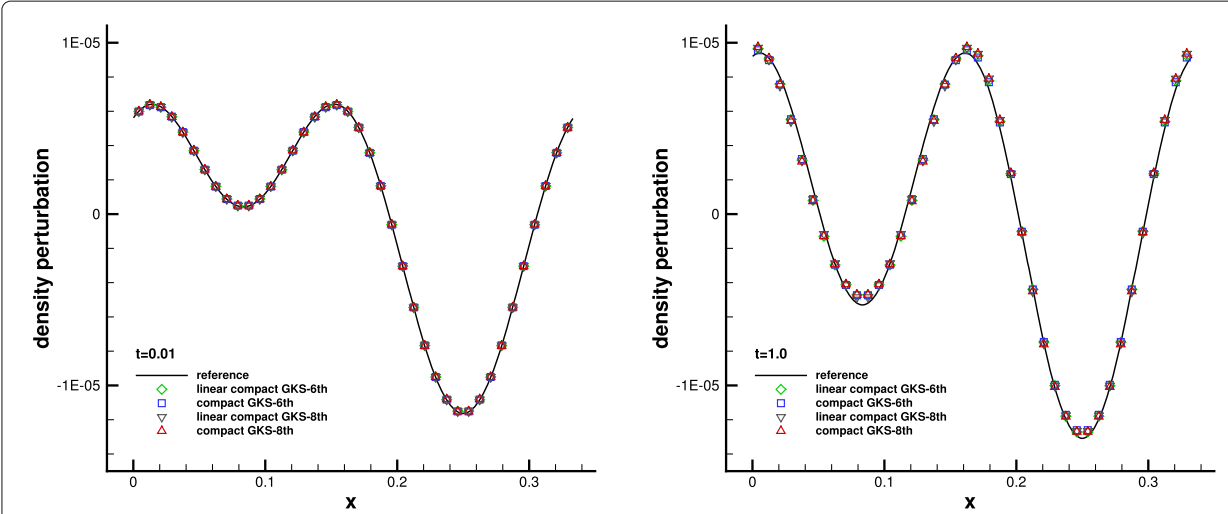

Fig. 9 One-dimensional acoustic problem: the distributions of density perturbation obtained by compact GKS at $t=0.01$ (left, 1 period) and $t=1.0$ (right, 100 periods) with 40 mesh points

\subsection{One-dimensional shock-velocity variation interaction}

The interaction between strong shock wave and turbulence is an important flow problem in gas dynamics, where the shock passes across a local wave packet with a large variation of flow velocity. To validate the current schemes for this kind of problems, we propose a test of shock with a Mach number $M a=10$ crossing a velocity variation. The initial condition is given as follows

$$
(\rho, U, p)= \begin{cases}(8,8.25,116.5), & x \leq 1 \\ (1.4,0.2 \sin (2 \pi(x-1)), 1), & 1<x \leq 10 .\end{cases}
$$

The computational domain is $[0,10]$. In order to compare the resolution for capturing the multi-scale structure from different schemes, a relative coarse mesh with 400 points is used in the computation. The non-reflecting boundary condition is used at both ends. The computed density profiles and the local enlargement from different schemes at $t=$ 0.8 are shown in Fig. 10, and the distributions of velocity and its local enlargement are shown in Fig. 11. The current compact 6th-order and 8th-order schemes perform well for
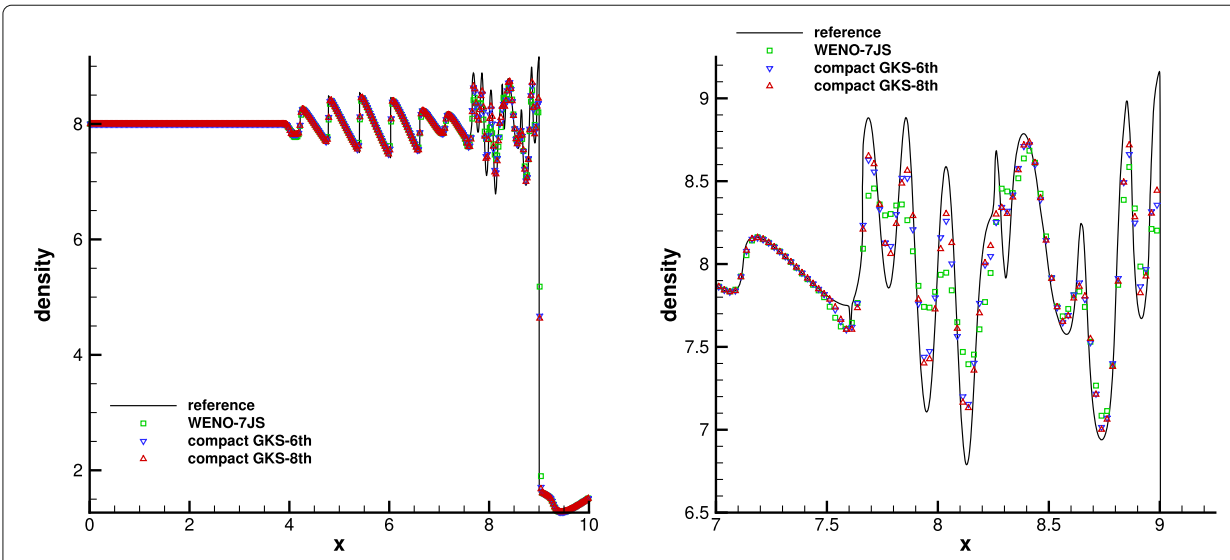

Fig. 10 One-dimensional shock crossing velocity perturbation: the density distribution and local enlargement from WENO-7JS non-compact GKS, 6th-order and 8th-order compact GKS at $t=0.8$ with 400 mesh points 

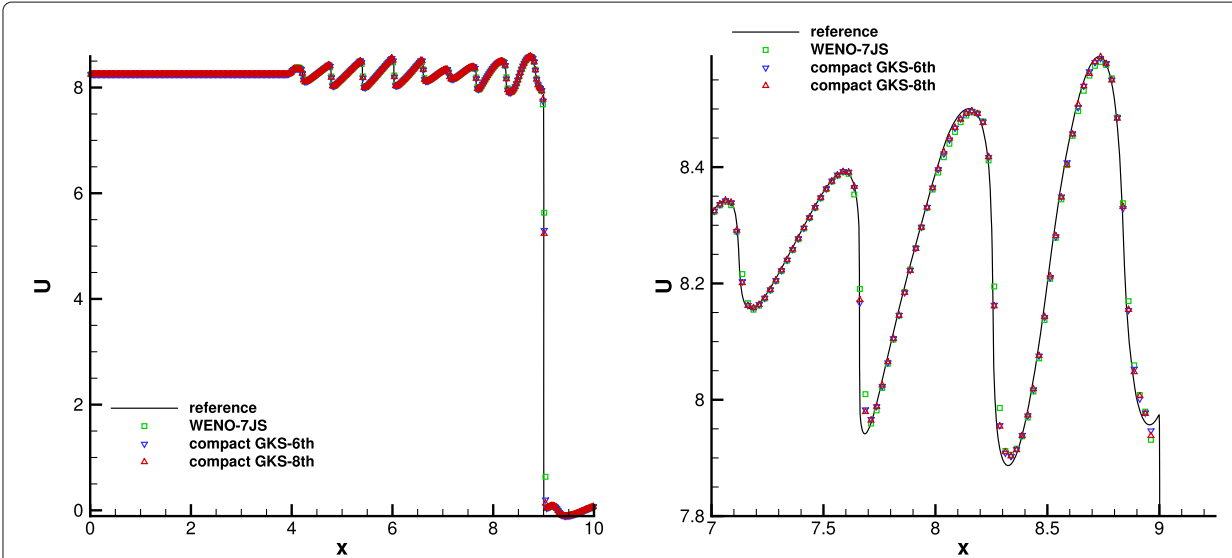

Fig. 11 One-dimensional shock crossing velocity perturbation: the velocity distribution and local enlargement from WENO-7JS non-compact GKS, 6th-order and 8th-order compact GKS at $t=0.8$ with 400 mesh points

resolving the multi-scale flow structures, where the non-compact WENO-7JS GKS has a relative large error. The compactness seems a preferred choice for a scheme with a better wave resolving power under strong shock condition.

\subsection{Double Mach reflection problem}

In this subsection, the double Mach reflection problem is tested. The test was extensively studied by Woodward and Colella [58] for the inviscid flow. The computational domain is $[0,4] \times[0,1]$, and a solid wall lies at the bottom of the computational domain starting from $x=1 / 6$. Initially a right-moving Mach 10 shock is positioned at $(x, y)=(1 / 6,0)$, which has a $60^{\circ}$ angle with the $\mathrm{x}$-axis. The initial pre-shock and post-shock conditions are

$$
\begin{aligned}
& (\rho, U, V, p)=(8,4.125 \sqrt{3},-4.125,116.5) \\
& (\rho, U, V, p)=(1.4,0,0,1)
\end{aligned}
$$

The reflecting boundary condition is used at the wall, and the exact post-shock condition is imposed for the rest of bottom boundary. At the top boundary, the flow variables are set to describe the exact motion of the Mach 10 shock. In this case, the compact 6th-order and 8th-order compact GKS are tested. The density distributions with $960 \times 240$ and $1920 \times 480$ uniform mesh points at $t=0.2$ are shown in Figs. 12 and 13, and the corresponding local density enlargements are shown in Fig. 14. The 6th-order and 8th-order schemes resolve the flow structure under the triple Mach stem very well.

\subsection{Two-dimensional Riemann problems}

In the following, the two-dimensional Riemann problems are considered. The first one is the interaction of four shocks $\overleftarrow{S_{21}} \overleftarrow{S_{32}} \overleftarrow{S_{41}} \overleftarrow{S_{34}}[59,60]$, where the backward shock waves connecting the areas $\Omega_{l}$ and $\Omega_{r}$ are denoted by $\overleftarrow{S_{l r}}$. The initial conditions are given as follows 

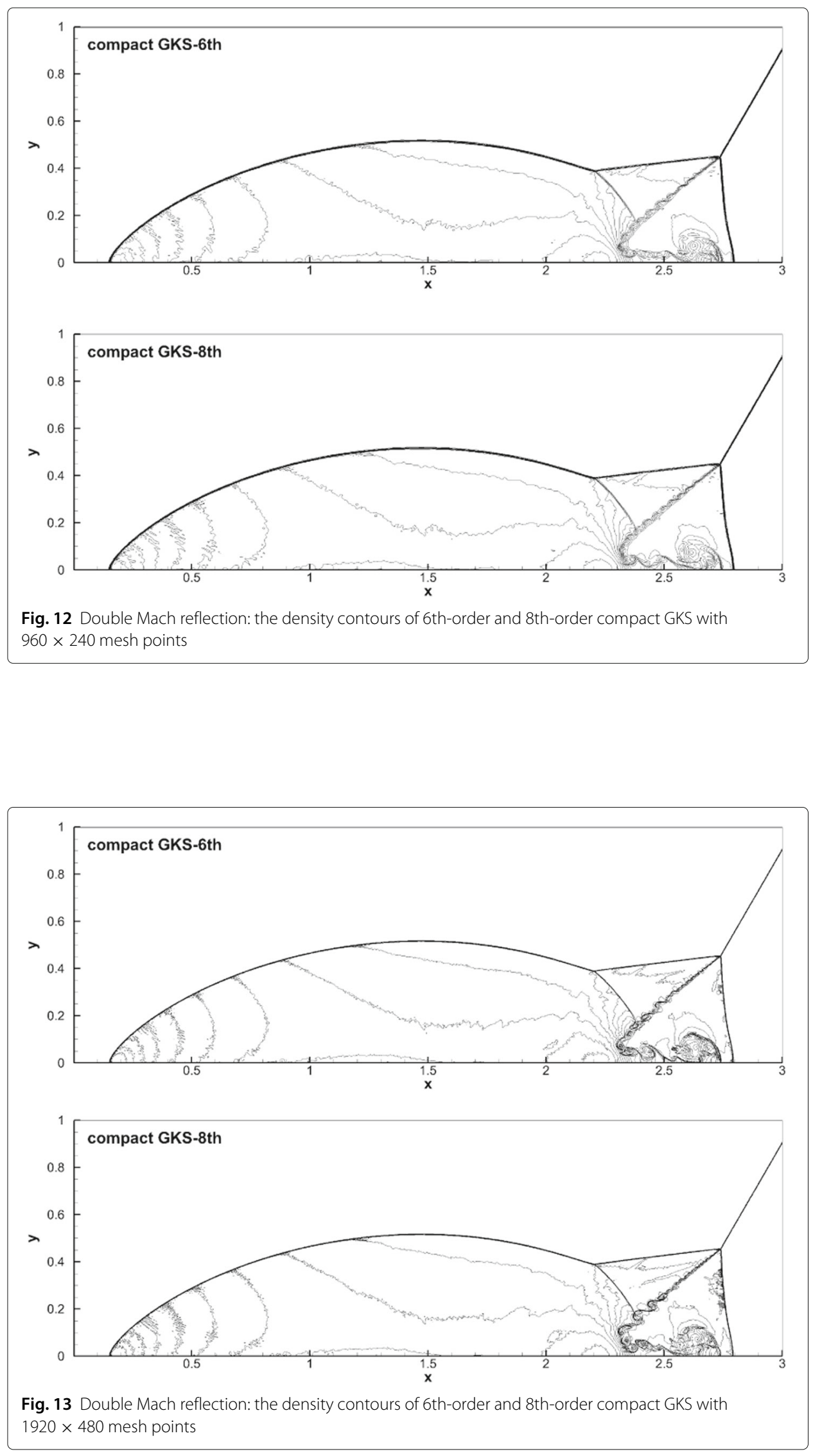

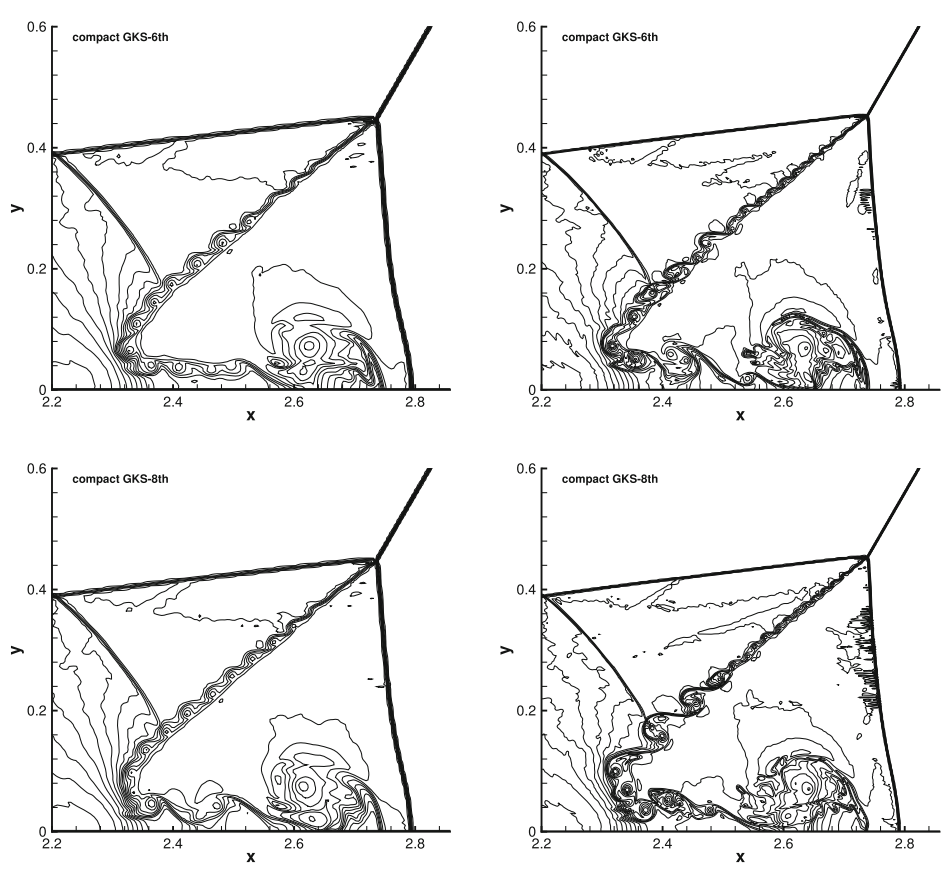

Fig. 14 Double Mach reflection: the local enlarged density distributions around the triple point of the 6th-order (up) and 8th-order (down) compact GKS with $960 \times 240$ (left) and $1920 \times 480$ (right) mesh points

$$
(\rho, U, V, p)= \begin{cases}(1.5,0,0,1.5), & \Omega_{1}: x>0.7, y>0.7 \\ (0.5323,1.206,0,0.3), & \Omega_{2}: x<0.7, y>0.7, \\ (0.138,1.206,1.206,0.029), & \Omega_{3}: x<0.7, y<0.7 \\ (0.5323,0,1.206,0.3), & \Omega_{4}: x>0.7, y<0.7 .\end{cases}
$$

This case is just the mathematical formation of the double Mach problem [58] and the symmetric line $x=y$ can be regarded as the rigid wall. The computational domain is $[0,1] \times[0,1]$, and the non-reflecting boundary conditions are used in all boundaries. The 6th-order and 8th-order compact GKS are used, and the numerical results are given in Fig. 15 at $t=0.4$ with $\Delta x=\Delta y=1 / 500$.

In the second case, the two-dimensional Riemann problem with four contact discontinuities $J_{21}^{-} J_{32}^{-} J_{41}^{-} J_{34}^{-}$is tested $[59,60]$, where the backward contact discontinuities connecting the areas $\Omega_{l}$ and $\Omega_{r}$ are denoted as $J_{l r}^{-}$. The initial conditions are given as follows

$$
(\rho, U, V, p)= \begin{cases}(1,0.75,-0.5,1), & \Omega_{1}: x>0.5, y>0.5 \\ (2,0.75,0.5,1), & \Omega_{2}: x<0.5, y>0.5 \\ (1,-0.75,0.5,1), & \Omega_{3}: x<0.5, y<0.5 \\ (3,-0.75,-0.5,1), & \Omega_{4}: x>0.5, y<0.5 .\end{cases}
$$

The computational domain is $[0,1] \times[0,1]$, and the non-reflecting boundary conditions are also used in all boundaries. The instantaneous interaction of the contact discontinuities results in entropy wave and vortex sheets. To obtain the detailed flow structure, the uniform mesh with $\Delta x=\Delta y=1 / 500$ are used. The numerical results from compact GKS are given in Fig. 16 at $t=0.5$. 

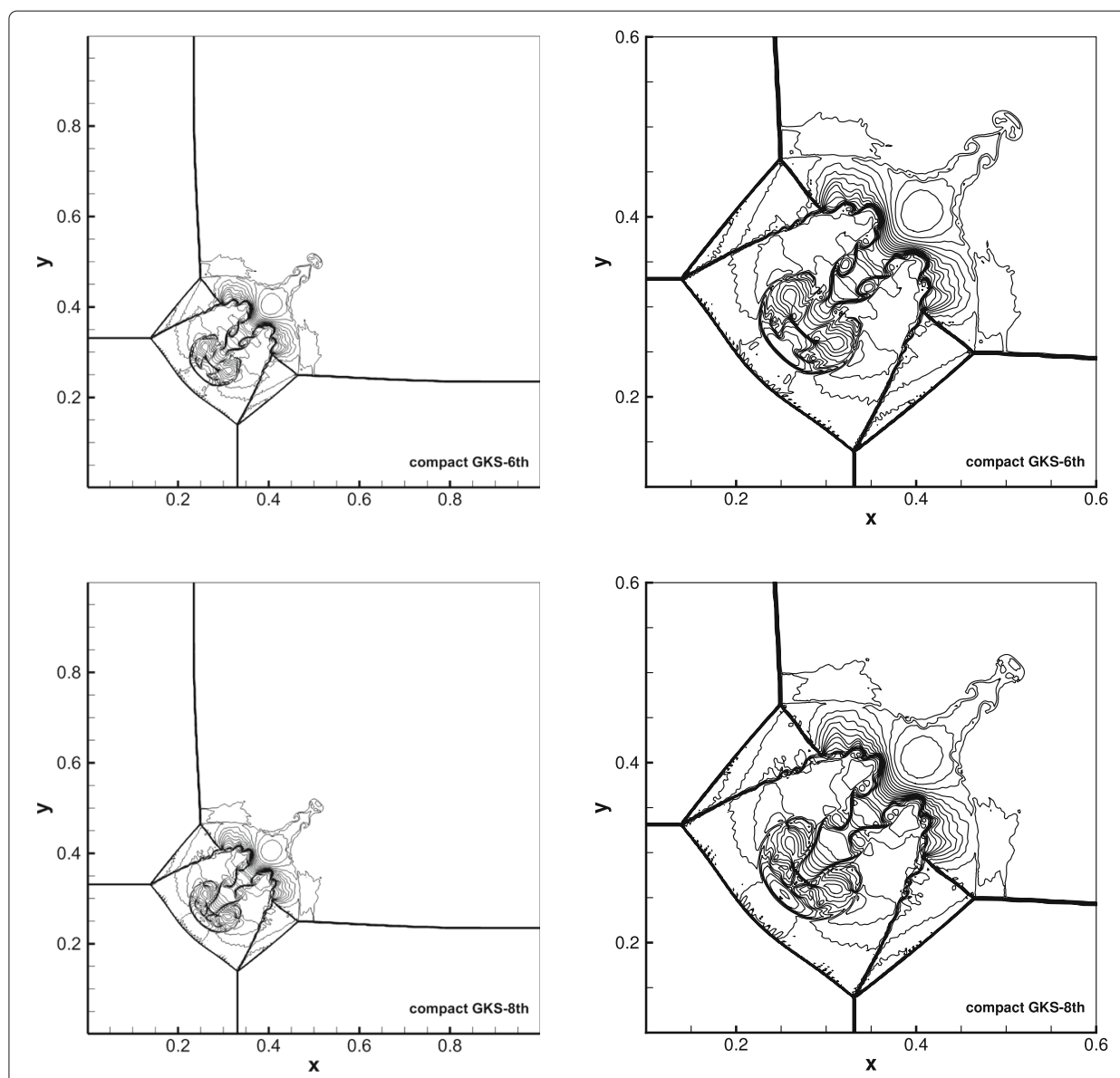

Fig. 15 2D Riemann problem: the density distributions by 6th-order and 8th-order compact GKS for the interaction of four shock waves at $t=0.4$ with $500 \times 500$ mesh points
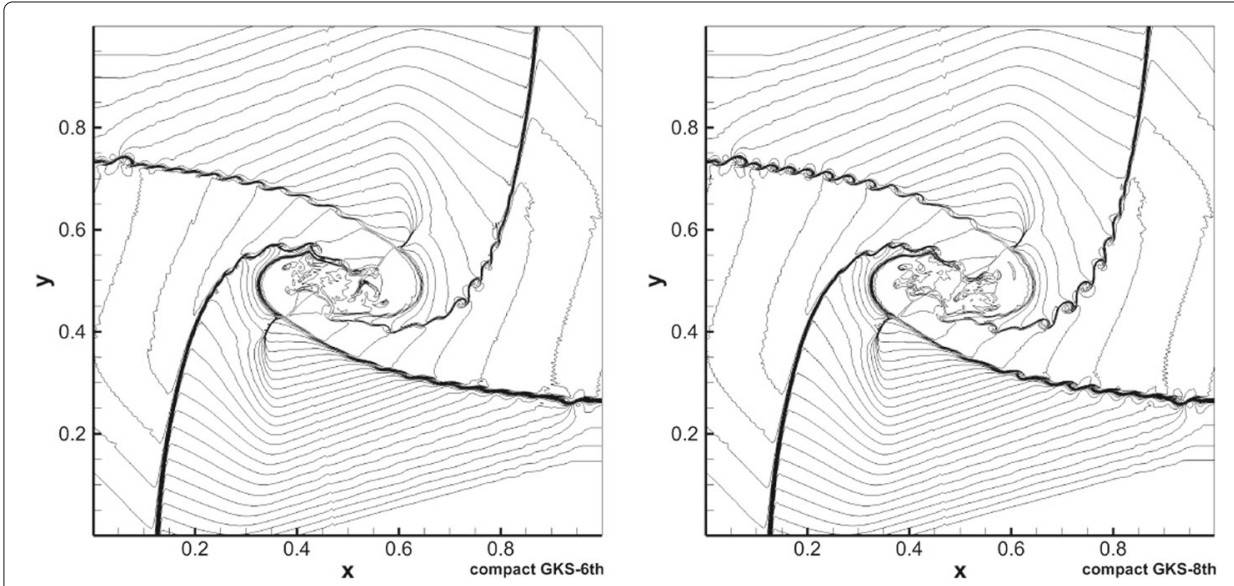

Fig. 16 2D Riemann problem: the density distributions from 6th-order and 8th-order compact GKS for the interactions of four contact discontinuities at $t=0.5$ with $500 \times 500$ mesh points 


\subsection{Lid-driven cavity flow}

Here we test the performance of the compact GKS for the capturing of viscous flow solutions. The lid-driven cavity problem is one of the most important benchmarks for validating incompressible Navier-Stokes flow solvers. The fluid is bounded by a unit square and is driven by a uniform translation of the top boundary. In this case, the flow is simulated with Mach number $M a=0.15$ and all boundaries are isothermal and nonslip. The computational domain $[0,1] \times[0,1]$ is covered with $65 \times 65$ mesh points. Numerical simulations are conducted for two different Reynolds numbers, i.e., $R e=1000$ and $R e=3200$. The streamlines corresponding to $R e=$ 1000 are shown in Fig. 17. The $U$-velocities along the center vertical line and $V$ velocities along the center horizontal line are shown in Fig. 18. The benchmark data [61] for $R e=1000$ and $R e=3200$ are also presented, and the simulation results match well with these benchmark data. The results from 8th-order compact GKS are almost identical to the reference solutions even for $R e=3200$ case. The cavity case fully validates the higher-order accuracy of compact GKS for viscous flow.

\subsection{Two-dimensional acoustic wave}

The case is an interaction of a shock wave with a single vortex as studied by Inoue and Hattori [62], where the fluid is viscous. The computational domain is $[-20,8] \times[-12,12]$. The initial counterclockwise vortex (the case $C$ in [62]) is set as follows

$$
U_{\theta}(r)=M_{\nu} r e^{\left(1-r^{2}\right) / 2}, \quad U_{r}=0,
$$

and the distribution of pressure and density upstream of shock are

$$
\begin{aligned}
& p(r)=\frac{1}{\gamma}\left[1-\frac{\gamma-1}{2} M_{\nu}^{2} e^{\left(1-r^{2}\right)}\right]^{\gamma /(\gamma-1)}, \\
& \rho(r)=\left[1-\frac{\gamma-1}{2} M_{\nu}^{2} e^{\left(1-r^{2}\right)}\right]^{1 /(\gamma-1)},
\end{aligned}
$$

where $U_{\theta}$ and $U_{r}$ are the tangential and radial velocity respectively. Mach number $M_{v}$ of the vortex is $M_{v}=0.25$. The Mach number of shock wave is $M_{s}=1.2$. The Reynolds number is $R e=800$ defined by $R e=\rho_{\infty} a_{\infty} / \mu_{\infty}$, where the subscript
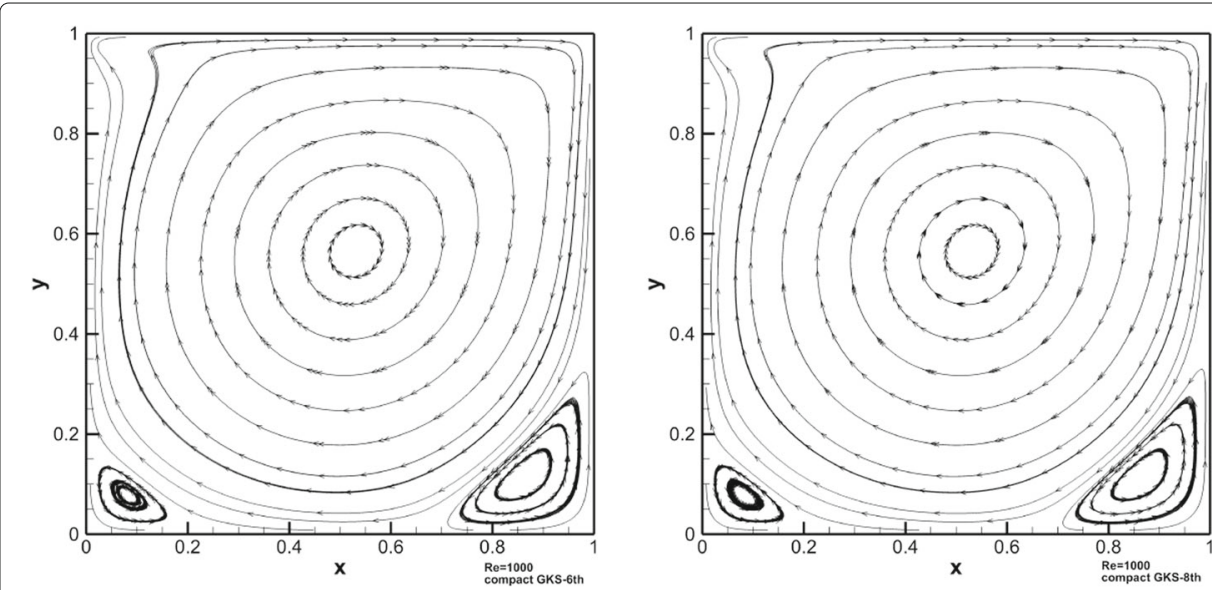

Fig. 17 Lid-driven cavity flow: streamlines with $65 \times 65$ uniform mesh points for $R e=1000$ by 6 th-order and 8th-order compact GKS 

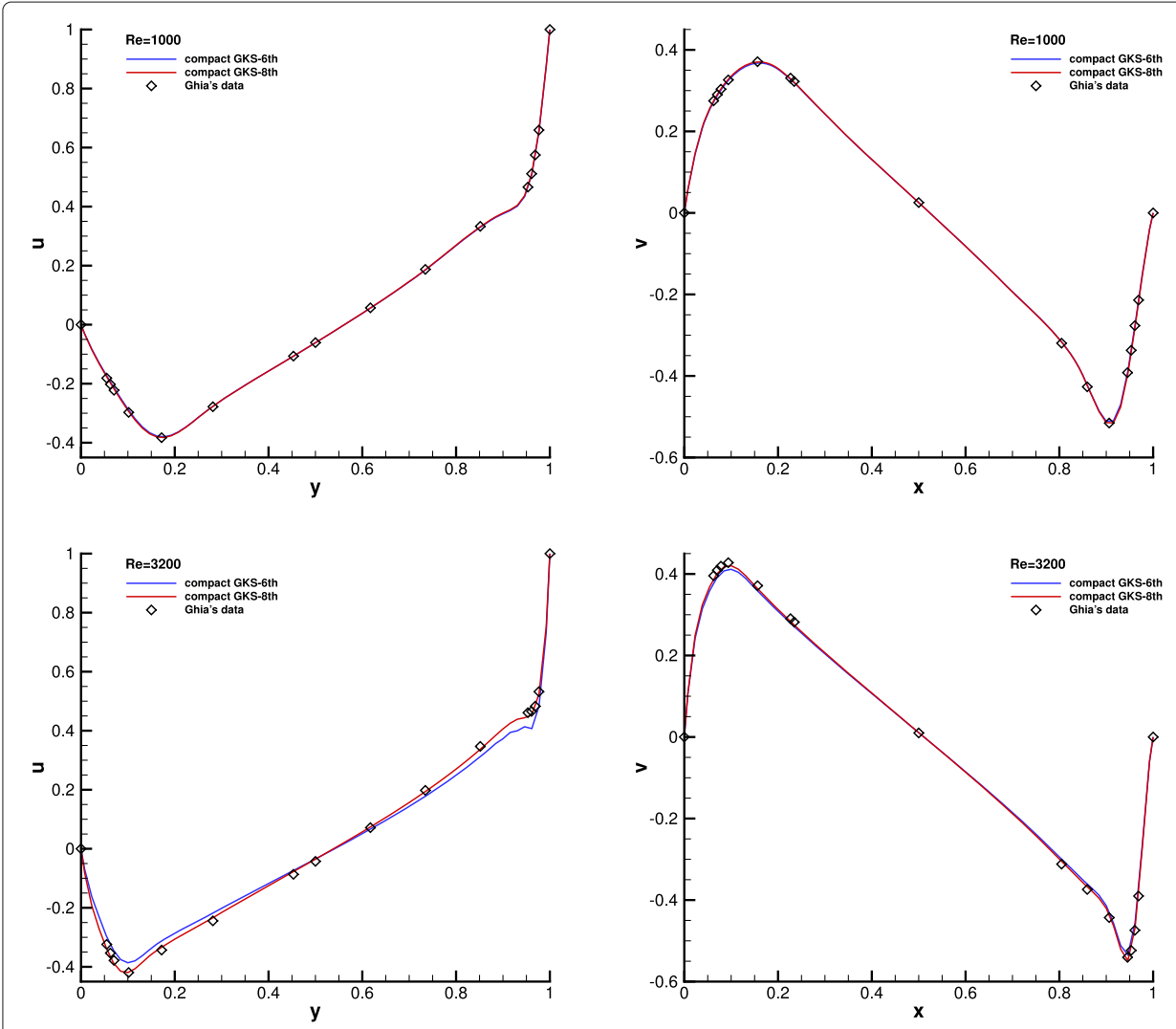

Fig. 18 Lid-driven cavity flow: U-velocity along vertical centerline and V-velocity along horizontal center-line with $65 \times 65$ uniform mesh points at $R e=1000$ and 3200

$\infty$ denotes the quantity downstream of the shock wave. The initial location of vortex is $\left(x_{v}, y_{v}\right)=(2,0)$, and the stationary shock is at $x=0$. In the computation, the supersonic inflow boundary conditions at $x=8$ as well as the periodic boundary conditions at $y= \pm 12$ are imposed. The non-reflecting boundary conditions are adopted at $x=-20$.

In the case, the compact 6th-order and 8th-order GKS are tested on the uniform mesh with $700 \times 600$ cells. The sound pressure at $t=6$ are shown in Fig. 19, where the sound pressure is defined as $\Delta p=\left(p-p_{\infty}\right) / p_{\infty}$. The pressure distribution in Fig. 19 clearly shows that the incident shock wave and two reflected shock waves are connected at the triple point, and the reflected shock waves emanate from the compression region of the incident shock wave. In addition, both the first and second sound waves have the clear quadruple nature with opposite sign, which is similar with the result in [62]. Both the 6th-order and 8th-order GKS perform well to resolve the shock wave and density wave, and the solution is sufficiently smooth to resolve small amplitude waves. The radial distribution of the sound pressure $\Delta p$ obtained by 6th-order and 8th-order compact GKS at $t=6$ with $700 \times 600$ mesh points are shown in Fig. 20. The radial distribution of the sound pressure is subtracted from the vortex center with the angle $\theta=135$ degree with the positive $x$ direction. The vortex center at $t=6$ is approximately located at $(-3.9,0.0)$. The results are concordant well with the results in [62], where the reference solution in [62] is obtained with $1044 \times 1170$ normal mesh points and 

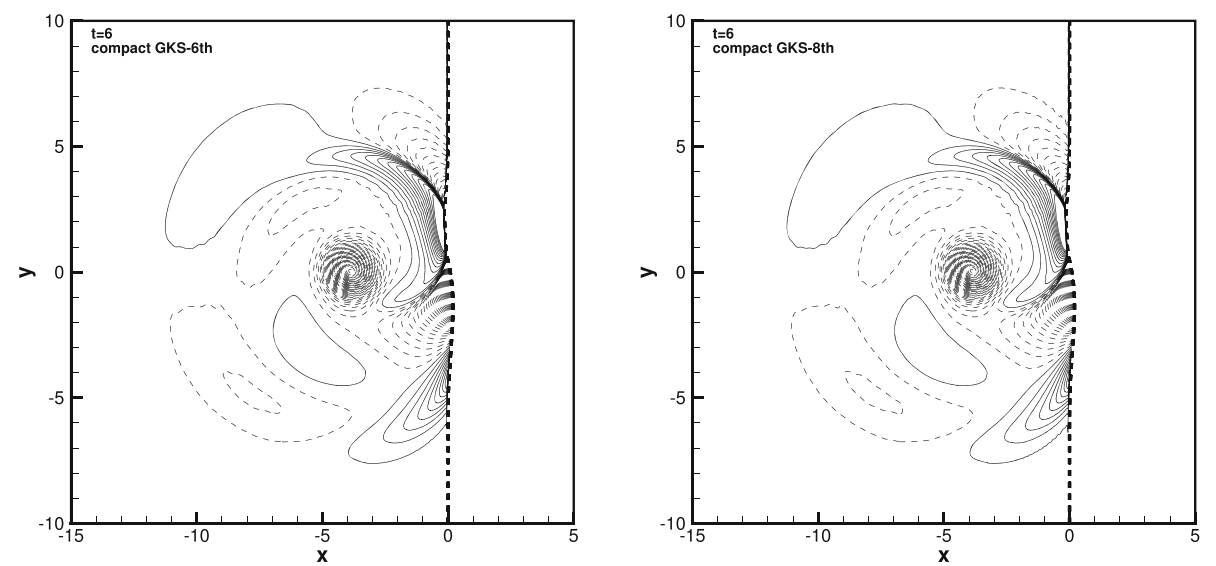

Fig. 19 Two-dimensional acoustic problem: sound pressure contours obtained by 6th-order and 8th-order compact GKS at $t=6$ with $700 \times 600$ mesh points. Shock and vortex Mach numbers are $M_{s}=1.2$ and $M_{v}=1$. Here 150 equal-spaced sound pressure contours from -0.5 to 0.05 are plotted. The dash line represents rarefaction region, and the solid line represents the compression region

a local mesh refinement with approximate $1 / 10$ of normal cell size at the shock region.

\subsection{Double shear flow}

The double shear flow is a canonical test problem for a scheme's accuracy and resolution in incompressible flows. Brown and Minion [63] performed a systematic comparison in this test using a number of flow parameters, focusing on the solution from the effect of cell resolution. The initial flow is set as

$$
\begin{aligned}
& U(x, y)= \begin{cases}\tanh (k(y-0.25)), & y \leq 0.5, \\
\tanh (k(0.75-y)), & y>0.5,\end{cases} \\
& V(x, y)=\delta \sin (2 \pi x)
\end{aligned}
$$

and the distributions of pressure and density are

$$
\rho(x, y)=1, p(x, y)=\frac{\rho U^{2}}{M_{a}^{2} \gamma},
$$
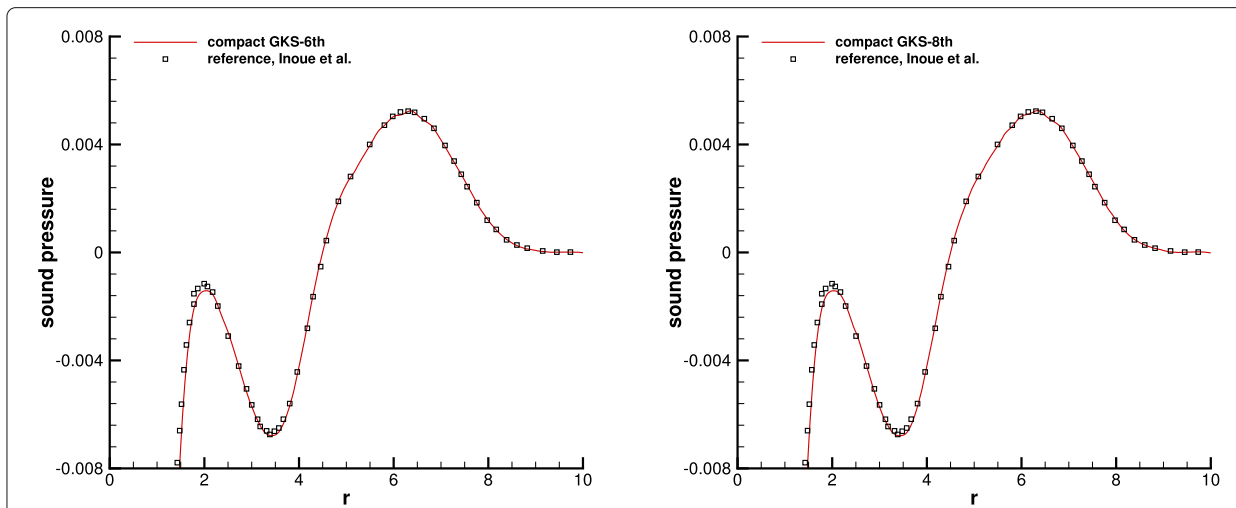

Fig. 20 Two-dimensional acoustic problem: radial distribution of the sound pressure $\Delta p$ obtained by 6th-order and 8th-order compact GKS at $t=6$ with $700 \times 600$ mesh points. The vortex center at $t=6$ is approximately located at $(-3.9,0.0)$ 
where $k=100$ and $\delta=0.05 . M_{a}$ is the Mach number, and $M_{a}=0.15$ is used in the following tests. The kinetic viscosity is $v=5.0 \times 10^{-5}$. The computational domain is $[0,1] \times[0,1]$, and the periodic boundary condition is adopted in both $x$ and $y$ directions.

The vorticity contours of linear and nonlinear compact GKS are shown in Figs. 21 and 22 respectively, where the linear and nonlinear WENO-5Z GKS is compared as well. Because of the high-order accuracy and high resolution of the compact GKS, the double shear layer can be exactly calculated on the coarse meshes used in [63]. In addition, the test with dynamic instability demonstrates the good stability of the compact GKS. Compared with the nonlinear 6th-order and 8th-order compact GKS, the linear compact GKS has better resolution. When refining the mesh, non-compact and compact GKS can give the same results in Fig. 23. The vorticity distribution and local enlargement at the line $x=0.5$ are shown in Fig. 24. The results obtained by linear WENO-5Z and linear compact GKS with $256 \times 256$ mesh points are presented in Fig. 24. The reference solution is obtained by linear 8th-order compact GKS with $512 \times 512$ mesh points. The linear compact GKS has better resolution than the linear non-compact WENO-5Z GKS. Besides, because of the compact reconstruction, the numerical solution of compact GKS has almost no spurious oscillation near the location with large variation of flow variable, such as at the location $y=0.13$ and $y=0.16$ in Fig. 24.

\section{Conclusion}

In this paper, a class of compact high-order gas-kinetic scheme with WENO reconstruction has been proposed for the compressible Euler and Navier-Stokes solutions. Based on the gas-kinetic theory, the current scheme depends solely on the time-accurate solution of a gas distribution function at a cell interface for the flux and conservative flow variable evaluation. With the update of pointwise values at cell interfaces, besides the cell-averaged conservative flow variables, their cell-averaged gradients can be updated as well. Therefore, the compact linear and nonlinear reconstructions can be obtained from the local cell averaged flow variables and their slopes. With symmetrical stencils, the 6th-order and 8th-order compact linear reconstructions are given and used in the 1-D Fourier analysis. The analysis elucidates spectral resolution of the current spatial discretization, even for very large wave number. The GKS flux makes the scheme be stable even for the initial reconstruction from symmetrical stencils. In
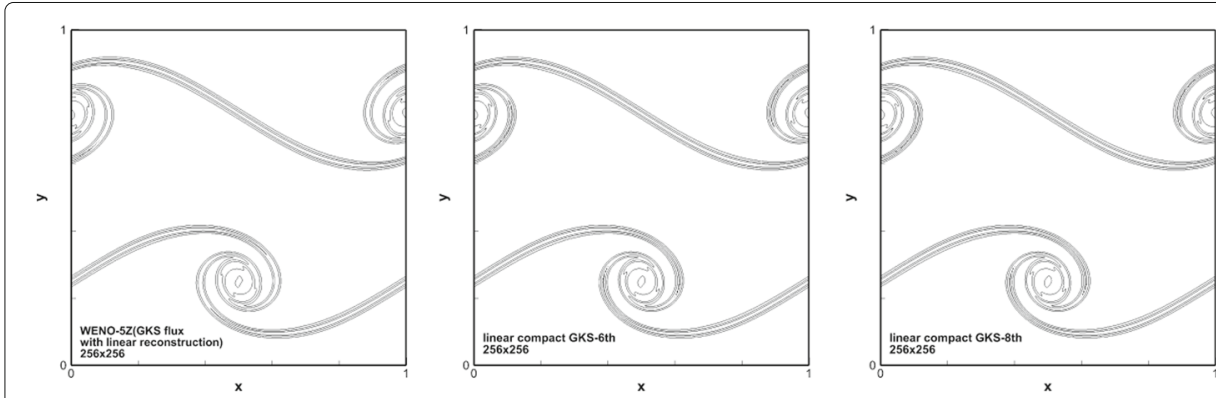

Fig. 21 Double shear flow: vorticity contours at $t=0.8$, obtained by non-compact linear reconstruction-based WENO-5th (GKS flux), and the linear 6th-order and 8th-order compact GKS with $256 \times 256$ mesh points 

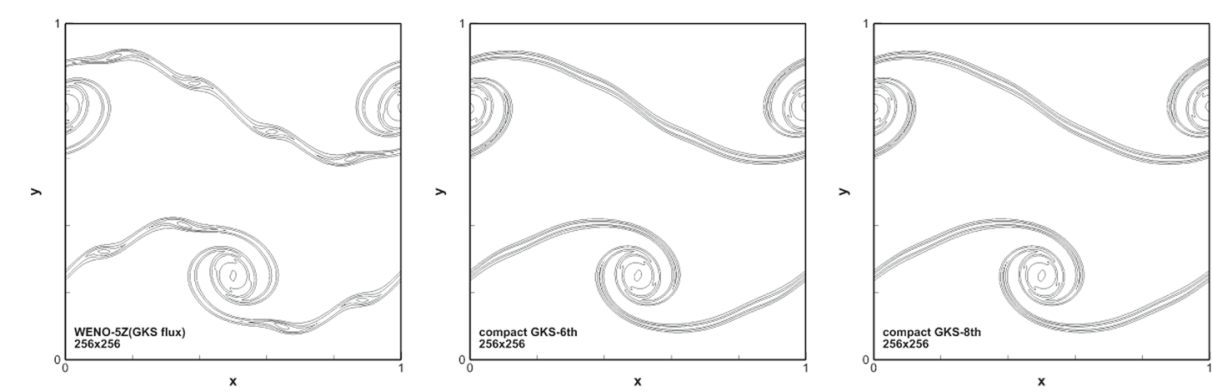

Fig. 22 Double shear flow: vorticity contours at $t=0.8$, obtained by non-compact nonlinear reconstruction-based WENO-5th(GKS flux), and the 6th-order and 8th-order compact GKS with $256 \times 256$ mesh points

order to capture shock and other discontinuities, the nonlinear compact WENO reconstruction has been constructed as well. Compared with other compact schemes, the current compact GKS is fully local and has explicit high-order discretization, without using any formulation for the globally connected flow variable and their slopes. Equipped with both linear and nonlinear compact reconstruction, the GKS gas evolution model can make a smooth transition dynamically from the initial nonlinear reconstruction for the non-equilibrium state to the final linear reconstruction for the equilibrium state. The transition rate depends on the local flow conditions. As a result, the current compact high-order scheme can naturally capture both shock discontinuities and small amplitude acoustic waves in a single computation without using any trouble cell detection and additional limiters. Due to the existence of the time-derivative of the flux function, the multi-stage and multi-derivative time stepping technique has been applied here for achieving high-order temporal accuracy of the scheme. More specifically, the fourth-order time accuracy has been achieved through two stages. Both GKS and DG methods have the same compact stencils. But, the GKS uses a much large CFL number $(\geq 0.3)$ for the 6th- and 8th-order schemes, and it is much more robust in capturing shock discontinuity and is much more efficient in simulating NS equations than the DG method. The extensive tests in this paper clearly demonstrate that the current 6th-order and 8th-order compact schemes advance the development of high-order CFD methods to a new level of maturity. The success of the current compact schemes
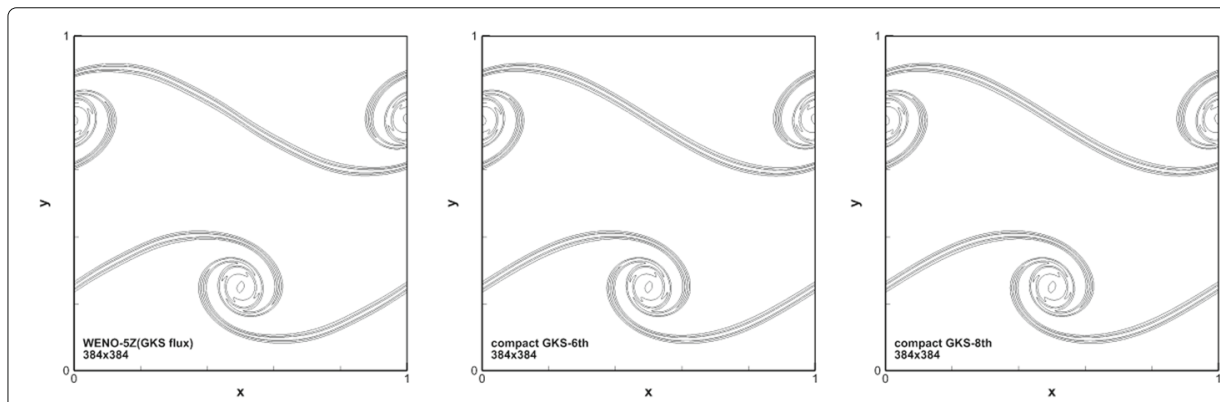

Fig. 23 Double shear flow: vorticity contours at $t=0.8$, obtained by non-compact nonlinear reconstruction-based WENO-5th(GKS flux), the 6th-order and 8th-order compact GKS with $384 \times 384$ mesh points 

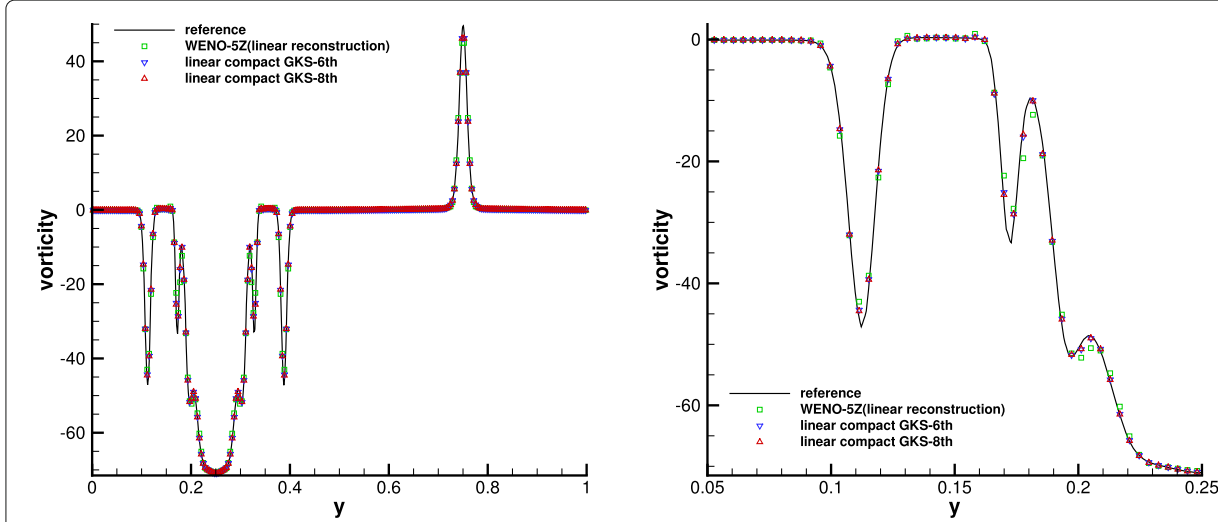

Fig. 24 Double shear flow: vorticity distribution and local enlargement at the line $x=0.5$ obtained by 5th-order upwind scheme, linear 6th-order and 8th-order compact GKS at $t=0.8$ with $256 \times 256$ mesh points. The reference solution is obtained by linear 8th-order compact GKS with $512 \times 512$ mesh points

depends solely on the high-order gas evolution model at a cell interface, which keeps and traces flow dynamics as close as possible to a real physical time evolution process. Any scheme based on the first order Riemann solver, which gives a time-independent gas evolution at a cell interface from two constant states whatever the high-order initial reconstruction is, may have difficulty to possess all these good properties of the current high-order GKS, such as the compactness, large CFL number, Navier-Stokes solutions, robustness, high efficiency, and the dynamic unification of linear and nonlinear reconstructions.

\section{Appendix}

For the WENO reconstruction, with the definition of smooth indicator in Eq. (31), the reconstructed polynomials from the sub-stencils for the determination of the left state of the cell interface $x_{i+1 / 2}$ are presented. Assume the candidate polynomial on a specific sub-stencil as

$$
w^{n}(x) \equiv \sum_{k=0}^{n} a_{k}^{n} x^{k} / k !
$$

where $n$ is the order of the polynomial and takes the values $n=2,3,4$ for the current 6th-order and 8th-order reconstruction schemes. Substituting $w^{n}(x)$ into the smooth indicator $\beta$ in Eq. (31), the result can be expressed by the coefficient $a_{k}^{n}$ as

$$
\begin{aligned}
\beta= & \left(a_{1}^{n} \cdot \Delta x\right)^{2}+\frac{13}{12}\left(a_{2}^{n} \cdot \Delta x^{2}\right)^{2}+\frac{1043}{960}\left(a_{3}^{n} \cdot \Delta x^{3}\right)^{2}+ \\
& \frac{35045}{32256}\left(a_{4}^{n} \cdot \Delta x^{4}\right)^{2}+\frac{1}{12}\left(a_{1}^{n} \cdot \Delta x\right) \cdot\left(a_{3}^{n} \cdot \Delta x^{3}\right)+\frac{7}{80}\left(a_{2}^{n} \cdot \Delta x^{2}\right) \cdot\left(a_{4}^{n} \cdot \Delta x^{4}\right) .
\end{aligned}
$$

For $n=2, a_{3}^{n}=a_{4}^{n}=0$, and for $n=3, a_{4}^{n}=0$. Therefore, once the candidate polynomial is given, the smoothing indicator can be fully determined. 
The polynomials of the sub-stencils for the 6th-order and 8th-order schemes are listed below,

$$
\begin{aligned}
w_{0}(x)= & \frac{1}{12}\left(W_{i-1}+11 W_{i}+\Delta x W_{i-1}^{\prime}\right)+\frac{1}{\Delta x}\left(-2 W_{i-1}+2 W_{i}-\Delta x W_{i-1}^{\prime}\right) \cdot x-\frac{2}{\Delta x^{2}}\left(W_{i-1}-W_{i}+\Delta x W_{i-1}^{\prime}\right) \cdot \frac{x^{2}}{2}, \\
\widetilde{w}_{1}(x)= & \frac{1}{24}\left(-W_{i-1}+26 W_{i}-W_{i+1}\right)+\frac{1}{16 \Delta x}\left(-23 W_{i-1}+20 W_{i}+3 W_{i+1}-10 \Delta x W_{i-1}^{\prime}\right) \cdot x+ \\
& \frac{1}{\Delta x^{2}}\left(W_{i-1}-2 W_{i}+W_{i+1}\right) \cdot \frac{x^{2}}{2}+\frac{3}{2 \Delta x^{3}}\left(3 W_{i-1}-4 W_{i}+W_{i+1}+2 \Delta x W_{i-1}^{\prime}\right) \cdot \frac{x^{3}}{6}, \\
w_{2}(x)= & \frac{1}{48}\left(43 W_{i}+16 W_{i+1}-11 W_{i+2}+6 \Delta x W_{i+2}^{\prime}\right)+\frac{1}{16 \Delta x}\left(-31 W_{i}+60 W_{i+1}-29 W_{i+2}+14 \Delta x W_{i+2}^{\prime}\right) \cdot x+ \\
& \frac{1}{2 \Delta x^{2}}\left(5 W_{i}-16 W_{i+1}+11 W_{i+2}-6 \Delta x W_{i+2}^{\prime}\right) \cdot \frac{x^{2}}{2}+\frac{3}{2 \Delta x^{3}}\left(-W_{i}+4 W_{i+1}-3 W_{i+2}+2 \Delta x W_{i+2}^{\prime}\right) \cdot \frac{x^{3}}{6}, \\
w_{3}(x)= & \frac{1}{24}\left(-W_{i-1}+26 W_{i}-W_{i+1}\right)+\frac{1}{2 \Delta x}\left(-W_{i-1}+W_{i+1}\right) \cdot x+\frac{1}{\Delta x^{2}}\left(W_{i-1}-2 W_{i}+W_{i+1}\right) \cdot \frac{x^{2}}{2}, \\
w_{4}(x)= & \frac{1}{24}\left(23 W_{i}+2 W_{i+1}-W_{i+2}\right)+\frac{1}{2 \Delta x}\left(-3 W_{i}+4 W_{i+1}-W_{i+2}\right) \cdot x+\frac{1}{\Delta x^{2}}\left(W_{i}-2 W_{i+1}+W_{i+2}\right) \cdot \frac{x^{2}}{2}, \\
w_{5}(x)= & \frac{1}{960}\left(-175 W_{i-1}+1148 W_{i}-13 W_{i+1}-54 \Delta x W_{i-1}^{\prime}-108 \Delta x W_{i}^{\prime}\right)+\frac{1}{8 \Delta x}\left(W_{i-1}-W_{i+1}+10 \Delta x W_{i}^{\prime}\right) \cdot x+ \\
& \frac{1}{4 \Delta x^{2}}\left(19 W_{i-1}-20 W_{i}+W_{i+1}+6 \Delta x W_{i-1}^{\prime}+12 \Delta x W_{i}^{\prime}\right) \cdot \frac{x^{2}}{2}-\frac{3}{\Delta x^{3}}\left(W_{i-1}-W_{i+1}+2 \Delta x W_{i}^{\prime}\right) \cdot \frac{x^{3}}{6}- \\
& \frac{6}{\Delta x^{4}}\left(5 W_{i-1}-4 W_{i}-W_{i+1}+2 \Delta x W_{i-1}^{\prime}+4 \Delta x W_{i}^{\prime}\right) \cdot \frac{x^{4}}{24}, \\
w_{6}(x)= & \frac{1}{960}\left(707 W_{i}-292 W_{i+1}+545 W_{i+2}-612 \Delta x W_{i+1}^{\prime}-186 \Delta x W_{i+2}^{\prime}\right)+ \\
& \frac{1}{8 \Delta x}\left(-21 W_{i}+8 W_{i+1}+13 W_{i+2}-22 \Delta x W_{i+1}^{\prime}-4 \Delta x W_{i+2}^{\prime}\right) \cdot x+ \\
& \frac{1}{4 \Delta x^{2}}\left(25 W_{i}+28 W_{i+1}-53 W_{i+2}+60 \Delta x W_{i+1}^{\prime}+18 \Delta x W_{i+2}^{\prime}\right) \cdot \frac{x^{2}}{2}- \\
& \frac{3}{\Delta x^{3}}\left(3 W_{i}+8 W_{i+1}-11 W_{i+2}+10 \Delta x W_{i+1}^{\prime}+4 \Delta x W_{i+2}^{\prime}\right) \cdot \frac{x^{3}}{6} \\
& \frac{6}{\Delta x^{4}}\left(W_{i}+4 W_{i+1}-5 W_{i+2}+4 \Delta x W_{i+1}^{\prime}+2 \Delta x W_{i+2}^{\prime}\right) \cdot \frac{x^{4}}{24} \cdot
\end{aligned}
$$

\section{Acknowledgements}

The authors would like to thank our collaborators on the development of high-order gas-kinetic schemes in the past many years.

\section{Funding}

The current research is supported by Hong Kong research grant council (16206617) and National Science Foundation of China (11772281, 91852114, 11701038).

\section{Availability of data and materials}

All data and materials are available upon request.

\section{Availability of supporting data}

Not applicable.

\section{Authors' contributions}

Our group has been working on the topic for a long time. The research output is coming from our joint effort. All authors read and approved the final manuscript.

\section{Competing interests}

The authors declare that they have no competing interests.

\section{Author details}

${ }^{1}$ Department of Mechanical and Aerospace Engineering, Hong Kong University of Science and Technology, Clear Water Bay, Kowloon, Hong Kong. ${ }^{2}$ Department of Mathematics, Hong Kong University of Science and Technology, Clear Water Bay, Kowloon, Hong Kong. ${ }^{3}$ Shenzhen Research Institute, Hong Kong University of Science and Technology, Shenzhen, China.

Received: 21 February 2019 Accepted: 21 May 2019

Published online: 06 June 2019 


\section{References}

1. Bai Z, Zhong X (2019) New very high-order upwind multi-layer compact (MLC) schemes with spectral-like resolution for flow simulations. J Comput Phys 378:63-109

2. Lele SK (1992) Compact finite difference schemes with spectral-like resolution. J Comput Phys 103(1):16-42

3. Mahesh K (1998) A family of high order finite difference schemes with good spectral resolution. J Comput Phys 145(1):332-358

4. Wang Q, Ren Y-X, Pan J, Li W (2017) Compact high order finite volume method on unstructured grids III: Variational reconstruction. J Comput Phys 337:1-26

5. Reed WH, Hill T (1973) Triangular mesh methods for the neutron transport equation. Los Alamos National Laboratory Report LA-UR-73-479

6. Cockburn B, Shu C-W (1989) TVB Runge-Kutta local projection discontinuous Galerkin finite element method for conservation laws. II. General framework. Math Comput 52(186):411-435

7. Cockburn B, Shu C-W (1998) The Runge-Kutta discontinuous Galerkin method for conservation laws V: Multidimensional systems. J Comput Phys 141(2):199-224

8. Huynh HT A flux reconstruction approach to high-order schemes including discontinuous Galerkin methods, 18th AIAA Computational Fluid Dynamics Conference, AIAA 2007-4079

9. Shu C-W (2016) High order WENO and DG methods for time-dependent convection-dominated PDEs: A brief survey of several recent developments. J Comput Phys 316:598-613

10. Harten A (1997) High resolution schemes for hyperbolic conservation laws. J Comput Phys 135(2):260-278

11. Harten A, Engquist B, Osher S, Chakravarthy SR (1987) Uniformly high order accurate essentially non-oscillatory schemes, III. J Comput Phys 71(2):231-303

12. Liu X-D, Osher S, Chan T (1994) Weighted essentially non-oscillatory schemes. J Comput Phys 115(1):200-212

13. Jiang G-S, Shu C-W (1996) Efficient implementation of weighted ENO schemes. J Comput Phys 126(1):202-228

14. Shu C-W (2009) High order weighted essentially nonoscillatory schemes for convection dominated problems. SIAM Rev 51(1):82-126

15. Hill DJ, Pullin DI (2004) Hybrid tuned center-difference-WENO method for large eddy simulations in the presence of strong shocks. J Comput Phys 194(2):435-450

16. Taylor EM, Wu M, Mart MP (2007) Optimization of nonlinear error for weighted essentially non-oscillatory methods in direct numerical simulations of compressible turbulence. J Comput Phys 223(1):384-397

17. Henrick AK, Aslam TD, Powers JM (2005) Mapped weighted essentially non-oscillatory schemes: achieving optimal order near critical points. J Comput Phys 207(2):542-567

18. Borges R, Carmona M, Costa B, Don WS (2008) An improved weighted essentially non-oscillatory scheme for hyperbolic conservation laws. J Comput Phys 227(6):3191-3211

19. Ren Y-X, Zhang H, et al (2003) A characteristic-wise hybrid compact-WENO scheme for solving hyperbolic conservation laws. J Comput Phys 192(2):365-386

20. Zhang L, Wei L, Lixin H, Xiaogang D, Hanxin Z (2012) A class of hybrid DG/FV methods for conservation laws I: Basic formulation and one-dimensional systems. J Comput Phys 231(4):1081-1103

21. Choi JJ (2015) Hybrid spectral difference/embedded finite volume method for conservation laws. J Comput Phys 295:285-306

22. Lax P, Wendroff B (1960) Systems of conservation laws. Commun Pure Appl Math 13(2):217-237

23. Ben-Artzi M, Falcovitz J (1984) A second-order Godunov-type scheme for compressible fluid dynamics. J Comput Phys 55(1):1-32

24. Ben-Artzi M, Li J, Warnecke G (2006) A direct Eulerian GRP scheme for compressible fluid flows. J Comput Phys 218(1):19-43

25. Ben-Artzi M, Li J (2007) Hyperbolic balance laws: Riemann invariants and the generalized Riemann problem. Numer Math 106(3):369-425

26. Toro EF (2013) Riemann solvers and numerical methods for fluid dynamics: a practical introduction. Springer-Verlag

27. Dumbser M, Balsara DS, Toro EF, Munz C-D (2008) A unified framework for the construction of one-step finite volume and discontinuous Galerkin schemes on unstructured meshes. J Comput Phys 227(18):8209-8253

28. Li J, Du Z (2016) A Two-Stage Fourth Order Time-Accurate Discretization for Lax-Wendroff Type Flow Solvers Hyperbolic, I., Conservation Laws. SIAM J Sci Comput 38(5):A3046—A3069

29. Li J (2019) Two-stage fourth order: temporal-spatial coupling in computational fluid dynamics (CFD). Adv Aerodyn. 1:3. https://doi.org/10.1186/s42774-019-0004-9

30. Xu K (2015) Direct modeling for computational fluid dynamics: construction and application of unified gas-kinetic schemes. World Scientific, Singapore

31. Xu K (2001) A gas-kinetic BGK scheme for the Navier-Stokes equations and its connection with artificial dissipation and Godunov method. J Comput Phys 171(1):289-335

32. Xu K, Huang J-C (2010) A unified gas-kinetic scheme for continuum and rarefied flows. J Comput Phys 229(20):7747-7764

33. Li J, Li Q, Xu K (2011) Comparison of the generalized Riemann solver and the gas-kinetic scheme for inviscid compressible flow simulations. J Comput Phys 230(12):5080-5099

34. Pan L, Xu K, Li Q, Li J (2016) An efficient and accurate two-stage fourth-order gas-kinetic scheme for the Euler and Navier-Stokes equations. J Comput Phys 326:197-221

35. Pan L, Cheng J, Wang S, Xu K (2017) A two-stage fourth-order gas-kinetic scheme for compressible multicomponent flows. Commun Comput Phys 22(4):1123-1149

36. Pan L, Li J, Xu K (2017) A few benchmark test cases for higher-order Euler solvers. Numer Math Theory Methods Appl 10(4):711-736

37. Hairer E, Wanner G (1973) Multistep-multistage-multiderivative methods for ordinary differential equations. Computing 11(3):287-303

38. Seal DC, Güc̈lü Y, Christlieb AJ (2014) High-order multiderivative time integrators for hyperbolic conservation laws. J Sci Comput 60(1):101-140 
39. Christlieb AJ, Gottlieb S, Grant Z, Seal DC (2016) Explicit strong stability preserving multistage two-derivative time-stepping schemes. J Sci Comput 68(3):914-942

40. Ji X, Zhao F, Shyy W, Xu K (2018) A family of high-order gas-kinetic schemes and its comparison with Riemann solver based high-order methods. J Comput Phys 356:150-173

41. Xu K (2002) A slope-update scheme for compressible flow simulation. J Comput Phys 178(1):252-259

42. Pan L, Xu K (2015) A compact third-order gas-kinetic scheme for compressible Euler and Navier-Stokes equations. Commun Comput Phys 18(4):985-1011

43. Pan L, Xu K (2016) A third-order compact gas-kinetic scheme on unstructured meshes for compressible Navier-Stokes solutions. J Comput Phys 318:327-348

44. Ji X, Pan L, Shyy W, Xu K (2018) A compact fourth-order gas-kinetic scheme for the Euler and Navier-Stokes equations. J Comput Phys 372:446-472

45. Qiu J, Shu C-W (2004) Hermite WENO schemes and their application as limiters for Runge-Kutta discontinuous Galerkin method: one-dimensional case. J Comput Phys 193(1):115-135

46. Qiu J, Shu C-W (2005) Hermite WENO schemes and their application as limiters for Runge-Kutta discontinuous Galerkin method II: Two dimensional case. Comput Fluids 34(6):642-663

47. Zhu J, Qiu J (2009) Hermite WENO schemes and their application as limiters for Runge-Kutta discontinuous Galerkin method, III: Unstructured meshes. J Sci Comput 39(2):293-321

48. Balsara DS, Altmann C, Munz C-D, Dumbser M (2007) A sub-cell based indicator for troubled zones in RKDG schemes and a novel class of hybrid RKDG+HWENO schemes. J Comput Phys 226(1):586-620

49. Luo H, Baum JD, Löhner R (2007) A Hermite WENO-based limiter for discontinuous Galerkin method on unstructured grids. J Comput Phys 225(1):686-713

50. Luo H, Xia Y, Li S, Nourgaliev R, Cai C (2012) A Hermite WENO reconstruction-based discontinuous Galerkin method for the Euler equations on tetrahedral grids. J Comput Phys 231(16):5489-5503

51. Luo H, Xia Y, Spiegel S, Nourgaliev R, Jiang Z (2013) A reconstructed discontinuous Galerkin method based on a hierarchical WENO reconstruction for compressible flows on tetrahedral grids. J Comput Phys 236:477-492

52. Bhatnagar PL, Gross EP, Krook M (1954) A model for collision processes in gases. I. Small amplitude processes in charged and neutral one-component systems. Phys Rev 94(3):511

53. Zhou G, Xu K, Liu F (2017) Simplification of the flux function for a high-order gas-kinetic evolution model. J Comput Phys 339:146-162

54. Chan RP, Tsai AY (2010) On explicit two-derivative Runge-Kutta methods. Numer Algoritm 53(2-3):171-194

55. Deng X, Zhang H (2000) Developing high-order weighted compact nonlinear schemes. J Comput Phys 165(1):22-44

56. Shu C-W, Osher S (1989) Efficient implementation of essentially non-oscillatory shock-capturing schemes, II. J Comput Phys 83(1):32-78

57. Titarev VA, Toro EF (2004) Finite-volume WENO schemes for three-dimensional conservation laws. J Comput Phys 201(1):238-260

58. Woodward P, Colella P (1984) The numerical simulation of two-dimensional fluid flow with strong shocks. J Comput Phys 54(1):115-173

59. Lax PD, Liu X-D (1998) Solution of two-dimensional Riemann problems of gas dynamics by positive schemes. SIAM J Sci Comput 19(2):319-340

60. Zhang T, Zheng YX (1990) Conjecture on the structure of solutions of the Riemann problem for two-dimensional gas dynamics systems. SIAM J Math Anal 21(3):593-630

61. Ghia U, Ghia KN, Shin C (1982) High-Re solutions for incompressible flow using the Navier-Stokes equations and a multigrid method. J Comput Phys 48(3):387-411

62. Inoue O, Hattori Y (1999) Sound generation by shock-vortex interactions. J Fluid Mech 380:81-116

63. Brown DL (1995) Performance of under-resolved two-dimensional incompressible flow simulations. J Comput Phys 122(1):165-183

\section{Publisher's Note}

Springer Nature remains neutral with regard to jurisdictional claims in published maps and institutional affiliations.

\section{Ready to submit your research? Choose BMC and benefit from:}

- fast, convenient online submission

- thorough peer review by experienced researchers in your field

- rapid publication on acceptance

- support for research data, including large and complex data types

- gold Open Access which fosters wider collaboration and increased citations

- maximum visibility for your research: over $100 \mathrm{M}$ website views per year

At $\mathrm{BMC}$, research is always in progress.

Learn more biomedcentral.com/submissions 